\title{
Instantons and Fermions in the Field of Instanton
}

\author{
A. S. Schwarz \\ Department of Theoretical Physics, Moscow Physical Engineering Institute, \\ Moscow M 409, USSR
}

\begin{abstract}
The number of instantons and the number of zero fermion modes in the field of instanton are calculated. The quantum fluctuations of instantons are studied.
\end{abstract}

\section{Section 1. Introduction}

Let us consider gauge fields taking values in the Lie algebra of simple compact non-abelian Lie group $G$. The topological number of the field $A_{\alpha}$ having finite euclidean Yang-Mills action $S=\frac{1}{2 g^{2}} \int\left\langle F_{\alpha \beta}, F^{\alpha \beta}\right\rangle d V$ can be defined as

$$
q=\frac{1}{16 \pi^{2}} \int\left\langle F_{\alpha \beta}, * F^{\alpha \beta}\right\rangle d V
$$

It is proved in [1] that $S \geqq 8 \pi^{2} g^{-2}|g|$ and $S=8 \pi^{2} g^{-2}|g|$ if

$$
F_{\alpha \beta}=* F_{\alpha \beta}
$$

for $q \geqq 0$

$$
F_{\alpha \beta}=-* F_{\alpha \beta}
$$

for $q \leqq 0$. Here $F_{\alpha \beta}$ denotes the strength of the field $A_{\alpha}$ and $* F_{\alpha \beta}$ the dual tensor. Following [2] we use the name instanton for solutions of the duality Eq. (2) and the name anti-instanton for solutions of (3). The Eqs. (2), (3) are conformally invariant and therefore we can replace the fields on the euclidean space by the fields on the sphere $S^{4}$ in (2), (3). The instantons having topological number 1 were found in [1] and used in many papers to understand the structure of quantum gauge theories. The quantum fluctuations of such instantons were studied in [2-4]. The examples of instantons having arbitrary topological number were given in [5-7]. G. 't Hooft found a 5q-parameter family of instantons having topological 
number $q$; it is shown in [7] that a slight modification of 't Hooft's construction gives a $(5 q+4)$-parameter family of instantons in the case $q \geqq 3$ and 13-parameter family in the case $q=2$.

We have shown in [8] that the solutions of (2) having topological number $q$ depend on $8 q-3$ parameters in the case $G=\mathrm{SU}(2)$. The proof is based on the Atiyah-Singer index theory; this theory was used in [8] to determine the number of zero fermion modes in the field of instanton too ${ }^{1}$.

After publication of [8] there appear many papers concerning the questions discussed in [8]. The essentially equivalent proof of main assertions of [8] was published in $[11]$. The considerations of the papers $[12,13]$ are also very near to those of [8]. They are based on the application of the index theory to the Dirac equation and on the connection between the Dirac equation and linearized duality equation (this connection was mentioned in [8] too). The zero fermions modes in the field of 't Hooft's instanton were found explicitly in [14] for fermions transforming according two-dimensional and three-dimensional representations of SU (2). The duality equation in the case of arbitrary gauge group was studied in [15] by means of methods of [8]. The relation between the local index theorem and Adler-Bell-Jackiv anomaly established in [8] was rediscovered in [16].

An important progress has been made in the paper [17], where the solution of duality equation was reduced to the problem of algebraic geometry. This problem is solved in [18-19].

There exists a clear and short review [20] of applications of topology and algebraic geometry to the study of instantons and more detailed reviews [21-23] of the same subject.

In the paper [24] we have studied the quantum fluctuations of instantons. The contribution of instantons in euclidean Green functions is expressed in [24] through regularized determinants of elliptic operators. To study the behaviour of this expression by conformal transformation of metric we apply the method used in [25] to prove the topological invariance of analytic torsion. The conformal properties of instanton determinants can be used to obtain information on twoinstanton contribution (only a factor depending on one parameter remains indetermined in this contribution).

As we show in separate papers $[26,27]$ the Ray-Singer torsion as well as instanton determinants can be expressed through the partition functions of degenerate quadratic Lagrangians. This physical interpretation permits to construct new topological invariants using the methods of quantum field theory.

In present paper we give the detailed proof of results of [8] and [24]. To facilitate the reading to physicists we have included in present paper the formulation of main mathematical results used in our proofs (Sect. 2, 3, 6, and Appendix I). Some results we have not found in the literature though they are perhaps well known. Most of results summarized in Sect. 2, 3, 6, and Appendix I are explained in many books and papers, but it seems that these books are not very easy for physicists. Of course the brief summary cannot replace detailed textbooks

1 In preliminary version [9] of the paper [8] we proved by means of index theory that the equation for infinitesimal variations of instanton (linearized duality equation) has at least $8 q-3$ independent solutions. In [10] this result was confirmed by means of direct calculation for 't Hooft's instantons 
on fibre space theory and theory of elliptic operators. However we hope that this summary will be useful by reading of this paper and other papers (for example [26-28]). We have defined some notions used in [26, 27] though they are not necessary in present paper.

In Sect. 4 we calculate the number of instantons. The Sect. 5 is devoted to zero fermion modes in the field of instantons. In Sect. 7 we use heuristic arguments to obtain the expression of instanton contribution through regularized determinants. In Sect. 8 we analyse the conformal properties of these determinants. In Sect. 9 we consider shortly the two-instanton determinants (see [29]). The results of Sect. 8 and 9 overlap partially with the results of paper [30].

All manifolds, maps, bundles, sections, forms under consideration will be supposed smooth.

\section{Section 2. Topological Preliminaries}

One says that a map $p$ of the space $E$ onto the space $B$ is a trivial fibration with the fibre $F$ if there exists such a homeomorphism $\varphi$ of $B \times F$ onto $E$ that $p \varphi(b, f)=b$ for every $b \in B, f \in F$. The map $\varphi$ is called a trivialization of the fibration $p$.

The map $p$ of the space $E$ onto the space $B$ is called a locally trivial fibration with a fibre $F$ if the space $B$ can be covered by open sets $U_{\alpha}$ in such a way that the map $p$ of $p^{-1}\left(U_{\alpha}\right)$ onto $U_{\alpha}$ is a trivial fibration, i.e. there exist homeomorphisms $\varphi_{\alpha}$ of $U_{\alpha} \times F$ onto $p^{-1}\left(U_{\alpha}\right)$ satisfying $p \varphi_{\alpha}(u, f)=u$. If $u \in U_{\alpha} \cap U_{\beta}$ we can determine a topological map $\varrho_{\alpha \beta}^{u}$ of $F$ onto $F$ by means of formula

$$
\varphi_{\beta}(u, f)=\varphi_{\alpha}\left(u, \varrho_{\alpha \beta}^{u} f\right) .
$$

The maps $\varrho_{\alpha \beta}^{u}$ will be called transition maps; one can say that the fibre space $E$ is glued from the direct products $U_{\alpha} \times F$ by means of maps $\varrho_{\alpha \beta}^{u}$. It is evident that for $u \in U_{\alpha} \cap U_{\beta} \cap U_{\gamma}$ we have

$$
\varrho_{\alpha \beta}^{u}=\varrho_{\alpha \beta}^{u} \varrho_{\beta \gamma}^{u} .
$$

If $F$ is a (complex) vector space and the transition maps $\varrho_{\alpha \beta}^{u}$ are linear we say that $\xi(E, B, F, p)$ is a (complex) vector bundle. If for every $u$ the map $\varrho_{\alpha \beta}^{u} \in G$, where $G$ is a subgroup of the group of linear transformations of $F$, we say that $\xi$ is a $G$-bundle. (We suppose that the trivializations $\varphi_{\alpha}$ are fixed.)

The continuous map $q$ of $B$ into $E$ will be called a section of $\xi$ if $p q(b)=b$ for every $b \in B$. If the fibre space is trivial then every section has a form $q(b)=\varphi(b, r(b))$ where $r$ is a map of $B$ into $F, \varphi$ is a trivialization. In general case $q(b)=\varphi_{\alpha}\left(b, r_{\alpha}(b)\right)$ where the maps $r_{\alpha}: U_{\alpha} \rightarrow F$ satisfy $r_{\alpha}(u)=\varrho_{\alpha \beta}^{u} r_{\beta}(u)$.

Let us consider a space $E$ and a topological group $G$, acting in $E$ on the right. If the group $G$ acts freely in $E$ (i.e. $e g \neq e$ if $e \in E, g \in G, g \neq 1$ ) and the identification map $p$ of $E$ onto coset space $B=E / G$ is a locally trivial fibration we say that the action of the group $G$ determines a principal fibration $\xi(E, B, G, p)$. (The last condition is always fulfilled if $G$ is a compact Lie group.) A principal fibration having a section $q$ is trivial; the trivialization is given by formula $\varphi(u, g)=q(u) g$. Let us consider such a covering $\left\{U_{\alpha}\right\}$ of the base $B=E / G$ that the fibration $\xi$ has a 
section $q_{\alpha}$ over $U_{\alpha}$; for every $\alpha$ the map $\varphi_{\alpha}(u, g)=q_{\alpha}(u) g$ determines a trivialization of $\xi$ over $U_{\alpha}$. We fix the covering $\left\{U_{\alpha}\right\}$ and the sections $q_{\alpha}$. It is evident that

$$
\varphi_{\alpha}(u, g)=q_{\alpha}(u) g=\varphi_{\beta}\left(u, \varrho_{\beta \alpha}^{u} g\right)=q_{\beta}(u)\left(\varrho_{\beta \alpha}^{u} g\right) \text {. }
$$

We define $\sigma_{\beta \alpha}^{u}$ as an element of the group $G$ satisfying

$$
q_{\alpha}(u)=q_{\beta}(u) \sigma_{\beta \alpha}^{u} .
$$

Then the transition map $\varrho_{\beta \alpha}^{u}$ transforms $g \in G$ into $\sigma_{\beta \alpha}^{u} g$.

Let us describe for example the tangential fibration of $n$-dimensional smooth manifold $M$. The space $E$ of this fibration consist of frames in $M$ (the set $\left(a_{1}, \ldots, a_{n}\right)$ of $n$ linearly independent vectors at the point of $M$ is called a frame at this point). It is easy to define a free action of the group GL $(n)$ on $E$ [a non-degenerate $n \times n$ matrix $g_{i j}$ transforms the frame $\left(a_{1}, \ldots, a_{n}\right)$ into the frame $\left(a_{1}^{\prime}, \ldots, a_{n}^{\prime}\right)$ where $\left.a_{i}^{\prime}=\sum a_{j} g_{j i}\right]$. This action determines the principal fibration $\zeta(E, M, \mathrm{GL}(n), p)$ which is called the tangential fibration of $B$. The local trivialization of $\zeta$ can be constructed by means of local coordinates in $M$; the transition maps assign to each point $b \in U_{\alpha} \cap U_{\beta}$ a Jacobian matrix $\left(\partial u_{(\alpha)}^{i} / \partial u_{(\beta)}^{j}\right)$ where $\left(u_{(\alpha)}^{1}, \ldots, u_{(\alpha)}^{n}\right)$ and $\left(u_{(\beta)}^{1}, \ldots, u_{(\beta)}^{n}\right)$ are local coordinates in $U_{\alpha}$ and $U_{\beta}$ respectively. If $M$ is a riemannian manifold one can give another definition of tangential fibration. It is convenient to consider in this case the space $E^{\prime}$ of orthonormal frames in $M$. The natural action of $O(n)$ in $E^{\prime}$ determines the principal fibration $\zeta^{\prime}\left(E^{\prime}, M, O(n), p^{\prime}\right)$ which is called a tangential fibration of riemannian manifold $M$.

For every representation $T$ of the group $G$ in the group of non-degenerate linear transformations of the vector space $F$ we define a vector bundle associated with the principal fibration $\xi(E, B, G, p)$ as a fibration $\xi_{T}\left(E_{T}, B, F, p_{T}\right)$ which is trivial over' $U_{\alpha}$ and has the transition maps $T\left(\varrho_{\alpha \beta}^{u}\right)$. One can give also an invariant definition of the associated vector bundle. Namely, the space $E_{T}$ can be obtained from $E \times F$ by means of identification $(e, f) \sim\left(e h, T\left(h^{-1}\right) f\right)$ where $h \in G$, the map $p_{T}$ is induced by the projection $(e, f) \rightarrow p(e)$.

Let us suppose that the base $B$ of vector bundle $\eta(E, B, F, p)$ is a smooth manifold. The space of smooth sections of the bundle $\eta$ can be considered as a linear space; this space will be denoted by $\Gamma(\eta)$. If the bundle $\eta$ is trivial the space $\Gamma(\eta)$ can be identified with the space of $F$-valued smooth functions on the base. If $\eta=\xi_{T}$ one can identify a section $f \in \Gamma\left(\xi_{T}\right)$ with a collection of $F$-valued functions $f_{\alpha}(u)$ defined for $u \in U_{\alpha}$ and satisfying

$$
f_{\alpha}(u)=T\left(\varrho_{\alpha \beta}^{u}\right) f_{\beta}(u)
$$

if $u \in U_{\alpha} \cap U_{\beta}$. One can say that the section $f \in \Gamma\left(\xi_{T}\right)$ can be considered as a function on the base taking values in the fibres of $\xi_{T}$. The exterior differential $p$-form on the base taking values in the fibres of $\xi_{T}$ can be defined as collection of forms

$$
\omega^{(\alpha)}=\frac{1}{p !} \omega_{\lambda_{1}, \ldots, \lambda_{p}}^{(\alpha)}(u) d u_{(\alpha)}^{\lambda_{1}} \wedge \ldots \wedge d u_{(\alpha)}^{\lambda_{p}}
$$

satisfying

$$
\omega_{\lambda_{1}, \ldots, \lambda_{p}}^{(\alpha)}(u)=\frac{\partial u_{(\beta)}^{\mu_{1}}}{\partial u_{(\alpha)}^{\lambda_{1}}} \ldots \frac{\partial u_{(\beta)}^{\mu_{p}}}{\partial u_{(\alpha)}^{\lambda_{p}}} T\left(\varrho_{\alpha \beta}^{u}\right) \omega_{\mu_{1}, \ldots, \mu_{p}}^{(\beta)}(u)
$$


if $u \in U_{\alpha} \cap U_{\beta}$ (here $\omega^{(\alpha)}$ is a $F$-valued $p$-form defined on $U_{\alpha}$, the local coordinates in $U_{\alpha}$ and $U_{\beta}$ are denoted by $\left(u_{(\alpha)}^{1}, \ldots, u_{(\alpha)}^{n}\right)$ and $\left(u_{(\beta)}^{1}, \ldots, u_{(\beta)}^{n}\right)$, the sign $\wedge$ denotes the exterior product: $\left.d u^{i} \wedge d u^{j}=-d u^{j} \wedge d u^{i}\right)$. The space of $p$-forms taking values in the fibres of $\xi_{T}$ will be denoted by $\Gamma^{p}\left(\xi_{T}\right)$; it is evident that $\Gamma^{0}\left(\xi_{T}\right)=\Gamma\left(\xi_{T}\right)$. It is easy to construct a vector bundle $\xi_{T}^{p}$ in such a way that sections of this bundle can be identified with $p$-forms taking values in the fibres of $\xi_{T}$ [i.e. $\left.\Gamma\left(\xi_{T}^{p}\right)=\Gamma^{p}\left(\xi_{T}\right)\right]$.

Let us consider the open set $U$ in the euclidean space $\mathscr{E}^{n}$, the trivial principal fibration $\xi(E, U, G, p)$ and the trivialization $\varphi: U \times G \rightarrow E$ of this fibration. The vector field $A_{\mu}$ defined on $U$ and taking values in the Lie algebra $\mathscr{G}$ of the group $G$ can be considered as a gauge field. Let $K$ denote a curve in $U$ with beginning point $\alpha(K)$ and ending point $\beta(K)$. The $T$-exponent $T \exp \left(-\int_{K} A_{\mu} d x^{\mu}\right)$ will be denoted by $h_{K}$. If the curve $K$ consists of two curves $K_{1}, K_{2}$ and $\alpha\left(K_{1}\right)=\alpha(K), \beta\left(K_{1}\right)=\alpha\left(K_{2}\right)$, $\beta\left(K_{2}\right)=\beta(K)$ then $h_{K}=h_{K_{2}} h_{K_{1}}$; for infinitesimal curve $h_{K}=1-A_{\mu} d x^{\mu}$. For every curve $K \subset U$ one can define a map $b_{K}$ of the fibre over the beginning point $\alpha(K)$ onto the fibre over the ending point $\beta(K)$ transforming the point $r=\varphi(\alpha(K), g)$ into the point $b_{K} r=\varphi\left(\beta(K), h_{K} g\right)$. Of course $b_{K}(r h)=b_{K}(r) h$ for every $h \in G$. One can say that the family of the maps $b_{K}$ determines the connection in the principal fibration $\xi$. The map $b_{K}$ can be interpreted as parallel transport of the fibre along the curve $K$. The gauge field $A_{\mu}$ determines also parallel transport along the curve $K \subset B$ of the field transforming according the representation $T$ of the group $G$ in the group of non-degenerate linear transformation of the space $F$. The vector $f_{1}$ over $\beta(K)$ is obtained by means of parallel transport from vector $f_{0}$ over $\alpha(K)$ if $f_{1}=T\left(h_{K}\right) f_{0}$. If $K(\lambda)$ is the set of points $\{u(\sigma)\}$ where $0 \leqq \sigma \leqq \lambda$ and $f_{\lambda}=T\left(h_{K(\lambda)}\right) f_{0}$ then $d f_{\lambda} / d \lambda=-t\left(A_{\mu}(u(\lambda)) f_{\lambda} d u^{\mu} / d \lambda\right.$, where $t$ denotes the representation of Lie algebra corresponding to $T$. If the field $f(u)$ satisfies

$$
\nabla_{\mu} f=\left(\partial_{\mu}+t\left(A_{\mu}\right)\right) f=0
$$

then it is unchanged by parallel transport. The transformation law of gauge field $A_{\mu}$ by the change of trivialization can be derived from the requirement that the maps $b_{K}$ do not depend from trivialization. We obtain that

$$
\begin{aligned}
h_{K}^{\prime} & =q(\beta(K)) h_{K} q^{-1}(\alpha(K)) \\
A_{\mu}^{\prime}(u) & =q(u) A_{\mu}(u) q^{-1}(u)-\left(\partial_{\mu} q\right) q^{-1}(u)
\end{aligned}
$$

if the trivializations $\varphi$ and $\varphi^{\prime}$ are related by the formula

$$
\varphi^{\prime}(u, g)=\varphi(u, q(u) g)
$$

We see that the fields $A_{\mu}$ and $A_{\mu}^{\prime}$ are gauge equivalent.

Let us suppose that $\xi(E, B, G, p)$ is a principal fibration and the base $B$ is $n$-dimensional smooth manifold. We denote by $\left\{U_{\alpha}\right\}$ the atlas of the manifold $B$ (i.e. we suppose that the manifold $B$ is covered by sets $U_{\alpha}$ and the coordinates $\left(u_{(\alpha)}^{1}, \ldots, u_{(\alpha)}^{n}\right)$ are introduced in $\left.U_{\alpha}\right)$. For every $\alpha$ we fix a section $q_{\alpha}$ of $\xi$ over $U_{\alpha}$. Corresponding trivialization of $\xi$ over $U_{\alpha}$ will be denoted by $\varphi_{\alpha}$ [i.e. $\left.\varphi_{\alpha}(u, g)=q_{\alpha}(u) g\right]$. The fibration $\xi$ is glued from trivial fibrations by means of transition maps $g \rightarrow \sigma_{\alpha \beta}^{u} g$. The gauge field $A$ in $\xi$ can be considered as a collection of 
fields $A_{\mu}^{(\alpha)}$ defined on $U_{\alpha}$, taking values in $\mathscr{G}$ and satisfying

$$
A_{\mu}^{(\alpha)}\left(u_{(\alpha)}^{1}, \ldots, u_{(\alpha)}^{n}\right)=\frac{\partial u_{(\alpha)}^{v}}{\partial u_{(\beta)}^{\mu}}\left(\sigma_{\beta \alpha}^{u} A_{v}^{(\beta)}\left(u_{(\beta)}^{1}, \ldots, u_{(\beta)}^{n}\right)\left(\sigma_{\beta \alpha}^{u}\right)^{-1}-\frac{\partial \sigma_{\beta \alpha}^{u}}{\partial u_{(\beta)}^{v}}\left(\sigma_{\beta \alpha}^{u}\right)^{-1}\right) .
$$

The gauge field $A$ determines the connection in $\xi$. Namely, if $K$ is a curve in $B$ one must divide $K$ onto curves $K_{1}, \ldots, K_{n}$ in such a way that each $K_{i}$ lies in one of $U_{\alpha}$ and define the map $b_{K}$ of the fibre over the beginning point of $K$ onto the fibre over the ending point of $K$ as $b_{K_{n}} \ldots b_{K_{1}}$ where $b_{K_{t}}$ are defined as earlier.

The transformation $\lambda$ of $E$ is called an automorphism of principal fibration $\xi(E, B, G, p)$ if $\lambda(e g)=\lambda(e g), p \lambda(e)=p(e)$ for $e \in E, g \in G$. It is easy to see that the automorphism of the trivial fibration can be represented in the form $\lambda \varphi(u, g)=\varphi(u, L(u) g)$ where $L(u)$ is a $G$-valued function on the base. In general case one can write that

$$
\lambda\left(\varphi_{\alpha}(u, g)\right)=\varphi_{\alpha}\left(u, L_{\alpha}(u) g\right)
$$

where

$$
u \in U_{\alpha}, \quad L_{\alpha}(u)=\sigma_{\alpha \beta}^{u} L_{\beta}(u)\left(\sigma_{\alpha \beta}^{u}\right)^{-1} \quad \text { if } \quad u \in U_{\alpha} \cap U_{\beta} .
$$

The automorphism $\lambda$ transforms the gauge field $A$ into the gauge field $A^{\prime}$ defined as a collection of fields

$$
A_{\mu}^{\prime(\alpha)}(u)=L_{\alpha}(u) A_{\mu}(u) L_{\alpha}^{-1}(u)-\left(\frac{\partial L_{\alpha}(u)}{\partial u_{(\alpha)}^{\mu}}\right) L_{\alpha}^{-1}(u) .
$$

It is easy to see that the maps $b_{K}=b_{K}^{A}$ and the maps $b_{K}^{\prime}=b_{K}^{A^{\prime}}$ are connected by the formula $b_{K}^{\prime}=\lambda b_{K} \lambda^{-1}$.

We say that the field $A^{\prime}$ is gauge equivalent to the field $A$ if there exists an automorphism transforming $A$ into $A^{\prime}$.

Let us consider the set $H_{e}$ of elements $c_{K} \in G$ satisfying $b_{K} e=e c_{K}$ [here $e \in E$ and $K$ is an arbitrary curve beginning and ending at $p(e)]$. It is easy to prove that $H_{e}$ is a group; this group is called a holonomy group at point $e$ [if $e=\varphi_{\alpha}(u, 1)$ then $H_{e}$ consists of elements $h_{K}^{(\alpha)}$ where $K$ run over the curves beginning and ending at $\left.u\right]$. The holonomy groups at different points of $E$ are conjugate. One can prove that the gauge field $A$ is gauge equivalent to the gauge field taking values in the subgroup $G^{\prime} \subset G$ if and only if there exists such a point that $H_{e} \subset G^{\prime}$.

The gauge field $A$ in a principal fibration $\xi$ determines a covariant differential $d_{A}$ in associated vector bundle $\xi_{T}$. The differential $d_{A}$ acts from $\Gamma^{p}\left(\xi_{T}\right)$ into $\Gamma^{p+1}\left(\xi_{T}\right)$; namely on $U_{\alpha}$ the operator $d_{A}$ transforms the form (1) in the form

$$
\left(d_{A} \omega\right)^{(\alpha)}=\frac{1}{p !} \nabla_{\varrho} \omega_{\lambda_{1}, \ldots, \lambda_{p}}^{(\alpha)} d u_{(\alpha)}^{\varrho} \wedge d u_{(\alpha)}^{\lambda_{1}} \wedge \ldots \wedge d u_{(\alpha)}^{\lambda_{\varrho}}
$$

where $\nabla_{\varrho}=\frac{\partial}{\partial u_{(\alpha)}^{Q}}+t\left(A_{\varrho}^{(\alpha)}\right)$ (here $t$ is the representation of the Lie algebra $\mathscr{G}$ corresponding to the representation $T$ of the Lie group $G$ ).

The sections of $\xi_{T}$ can be considered as fields transforming according the representation $T$ of $G$ and the covariant differential $d_{A}$ coincides with usual covariant differential of such fields. The gauge field determines the parallel transport of the fiber of vector bundle $\xi_{T}$ along the curve $K \subset B$. If the space $E_{T}$ is 
represented as space of pairs $(e, f), \quad e \in E, f \in F$ with identification $(e, f) \sim\left(e h, T\left(h^{-1}\right) f\right)$ then the parallel transport along $K$ transforms $(e, f)$ into $\left(b_{K} e, f\right)$. If the field $\psi$ transforming according the representation $T$ (i.e. the section of $\xi_{T}$ ) satisfies $d_{A} \psi=0$ then this field is unchanged by parallel transport and therefore for every $e \in E$ there exists such an element $f \in F, f \neq 0$ that the pairs $(e, f)$ and $\left(b_{K} e, f\right)=\left(e c_{K}, f\right)$ for every closed curve $K$ determine the same point of $E_{T}$. Using that $\left(e c_{K}, f\right) \sim\left(e, T\left(c_{K}^{-1}\right) f\right)$ we obtain that $T(g) f=f$ for every $g \in H_{e}$. If $G=\mathrm{SU}(2)$ and $T$ is an irreducible representation, we see that in the case under consideration the holonomy group is abelian and therefore the gauge field is gauge equivalent to a gauge field taking values in the abelian Lie algebra.

Let us consider the adjoint representation $\tau$ of the group $G$ in the Lie algebra $\mathscr{G}$ and corresponding associated vector bundle $\xi_{\tau}\left(E_{\tau}, B, \mathscr{G}, p_{\tau}\right)$. The strength of gauge field can be considered as collection of 2-forms

$$
F^{(\alpha)}=\frac{1}{2} F_{\mu \nu}^{(\alpha)} d u_{(\alpha)}^{\mu} \wedge d u_{(\alpha)}^{v},
$$

where

$$
F_{\mu \nu}^{(\alpha)}=\partial_{\mu} A_{v}^{(\alpha)}-\partial_{v} A_{\mu}^{(\alpha)}+\left[A_{\mu}^{(\alpha)}, A_{v}^{(\alpha)}\right] .
$$

It is easy to see that the forms $F^{(\alpha)}$ determine a 2-form taking values in the fibres of the bundle $\xi_{\tau}$. The difference of two gauge fields $\left(A_{\mu}^{(\alpha)}-\tilde{A}_{\mu}^{(\alpha)}\right) d u^{\mu}$ can be considered as a 1 -form taking values in the fibres of $\xi_{\tau}$. The infinitesimal automorphism of $\xi$ can be considered as a set of $\mathscr{G}$-valued functions $\omega^{(\alpha)}(u)$ satisfying $\omega^{(\alpha)}(u)$ $=\sigma_{\alpha \beta}^{u} \omega^{(\beta)}(u)\left(\sigma_{\alpha \beta}^{u}\right)^{-1}$; the functions $\omega^{(\alpha)}$ determine a section of $\xi_{\tau}$.

Let us suppose now that the vector bundle $\eta(E, B, F, p)$ is provided with hermitian structure [i.e. $\eta$ is an $U(m)$-bundle and $B$ is $n$-dimensional compact riemannian manifold]. In this case one can naturally define the scalar product in $\Gamma^{p}(\eta)$ and the operator $*$ acting from $\Gamma^{p}(\eta)$ into $\Gamma^{n-p}(\eta)$; the operator $*$ transforms the form (1) into the dual form

$$
\tilde{\omega}^{(\alpha)}=\frac{1}{(n-p) !} \tilde{\omega}_{\mu_{1}, \ldots, \mu_{n-p}}^{(\alpha)}(u) d u_{(\alpha)}^{\mu_{1}} \wedge \ldots \wedge d u_{(\alpha)}^{\mu_{n}-p},
$$

where

$$
\tilde{\omega}_{\mu_{1}, \ldots, \mu_{n-p}}^{\alpha}=\sqrt{g} \varepsilon_{\lambda_{1}, \ldots, \lambda_{p}, \mu_{1}, \ldots, \mu_{n-p}} \omega^{(\alpha) \lambda_{1}, \ldots, \lambda_{p}}
$$

(as usual $g=\operatorname{det} g_{\alpha \beta}, a_{\mu}=g_{\mu \nu} a^{v}, g_{\mu \nu}$ is the metric tensor). It is easy to check that the adjoint operator to the covariant differential $d_{A}$ is equal to $d_{A}^{*}=-* d_{A} *$ if the dimension of the manifold $M$ is even. The operator $d_{A}^{*}$ can be considered as covariant divergence; for example $d_{A}^{*}$ transforms the 1 -form $\omega_{\mu} d u^{\mu}$ into the 0 -form $-\nabla_{\mu} \omega^{\mu}$.

\section{Section 3. Elliptic Operators}

Let $A$ be a differential operator acting on vector-valued functions defined on bounded domain $D$ in euclidean space $\mathscr{E}^{n}$. The differential operator $A$ of order $N$ transforms the function $\left(f_{1}(u), \ldots, f_{m}(u)\right)$ into function $\left(f_{1}^{\prime}(u), \ldots, f_{m}^{\prime}(u)\right)$ where

$$
f_{i}^{\prime}(u)=\sum_{\left|\lambda_{1}+\ldots+\lambda_{n}\right| \leqq N} \sum_{1 \leqq i, j \leqq m} A_{i j}^{\lambda_{1}, \ldots, \lambda_{n}}(u) \partial_{1}^{\lambda_{1}} \ldots \partial_{n}^{\lambda_{n}} f_{j}(u)
$$


the coefficient functions $A_{i j}^{\lambda_{1}, \ldots, \lambda_{n}}(u)$ will be supposed smooth. The differential operator $A$ will be called an elliptic operator if for every $u \in D$ and for every $p \in \mathscr{E}^{n}$, $p \neq 0$ the symbol of this operator

$$
\sigma_{i j}(u, p)=\sum_{\left|\lambda_{1}+\ldots+\lambda_{n}\right|=N} A_{i j}^{\lambda_{1}, \ldots, \lambda_{n}} p_{1}^{\lambda_{1}} \ldots p_{n}^{\lambda_{n}}
$$

is a non-degenerate matrix. It is convenient to consider differential operators as operators in Sobolev spaces. The Sobolev space $W_{p}^{k}(D)$ is defined as completion of the space of smooth functions on $D$ with respect to the norm

$$
\|f\|_{(p)}^{(k)}=\sum_{\left|\lambda_{1}+\ldots+\lambda_{n}\right| \leqq k}\left\|\partial_{1}^{\lambda_{1}} \ldots \partial_{n}^{\lambda_{n}} f\right\|_{(p)}
$$

where $\|g\|_{(p)}$ denotes the $L^{p}$-norm:

$$
\|g\|_{(p)}=\left(\sum_{i}\left|g_{i}(u)\right|^{p} d^{n} u\right)^{1 / p} .
$$

The differential operator or order $N$ can be continuously extended on $W_{p}^{k}$ if $k \geqq N$ and acts from $W_{p}^{k}$ into $W_{p}^{k-N}$.

We will consider the differential elliptic operators acting from the space $\Gamma\left(\xi_{1}\right)$ into the space $\Gamma\left(\xi_{2}\right)$ where $\xi_{i}\left(E_{i}, M, F_{i}, p_{i}\right), i=1,2$, are vector bundles, $M$ is a compact manifold. (These operators can be defined as operators satisfying locally the requirements above.) If $A$ is such an operator and the bundles $\xi_{1}, \xi_{2}$ are provided with hermitian structure one can construct formally the adjoint operator $A^{*}$ acting from $\Gamma\left(\xi_{2}\right)$ into $\Gamma\left(\xi_{1}\right)$. It is easy to check that the operator $A^{*}$ is elliptic; $A^{*} A$ and $A A^{*}$ are non-negative self-adjoint elliptic operators acting in $\Gamma\left(\xi_{1}\right)$ and $\Gamma\left(\xi_{2}\right)$ respectively.

We denote by $l(A)$ the number of linearly independent solutions of equation $A f=0$, i.e. the number of zero modes of operator $A$. In other words $l(A)$ denotes the dimension of the kernel of $A$ :

$$
l(A)=\operatorname{dim} \operatorname{ker} A .
$$

(The kernel of $A$ is the space of solution of equation $A f=0$.) It is easy to verify that $l(A)=l\left(A^{*} A\right)$ and $l\left(A^{*}\right)=l\left(A A^{*}\right)$. The index of elliptic operator $A$ is defined by formula

$$
\text { index } A=l(A)-l\left(A^{*}\right)
$$

[the numbers $l(A)$ and $l\left(A^{*}\right)$ are finite therefore this definition is correct]. One can check that the index does not change by continuous variation ${ }^{2}$ of operator $A$; this assertion permits to use topological methods for calculation of index [31-33]. It follows from this fact that index $A$ does not depend on the choice of the hermitian structures in bundles $\xi_{1}, \xi_{2}$.

Let us consider a non-negative self-adjoint elliptic operator $K$ in the space $\Gamma(\xi)$ where $\xi(E, M, F, p)$ is provided with hermitian structure. Let $R$ be a differential operator of order zero (locally $R$ can be represented as operator of multiplication

2 One can say that operator depends continuously on parameter if the coefficient functions depend continuously on this parameter in the $C^{\infty}$-topology 
on matrix function). The asymptotics of $\operatorname{Sp} R \exp (-K t)$ for $t \rightarrow 0$ can be studied by semiclasical method. One can prove that for $t \rightarrow 0$

$$
\operatorname{Sp} R \exp (-K t) \approx \sum \Psi_{k}(R \mid K) t^{-k}
$$

where $k=\frac{n}{N}, \frac{n-1}{N}, \frac{n-2}{N}, \ldots, N$ is the order of $B$ and $n$ is the dimension of the base $M$. The coefficients can be calculated by means of Seeley formula [34], [33]. If $A$ is second order differential operator the coefficient $\Psi_{k}(R \mid K)$ can be represented as an integral over $M$ and the integrand is a rational function of coefficient functions of $R$ and derivatives of coefficient functions of $K$.

One can take $R=1$; then we obtain from (2) that for $t \rightarrow 0$

$$
\mathrm{Sp} \exp (-K t) \approx \sum \Phi_{k}(K) t^{-k}
$$

where

$$
\Phi_{k}(K)=\Psi_{k}(1 \mid K) \text {. }
$$

The index of elliptic operator $A$ can be represented in the form

$$
\text { index } A=\Phi_{0}\left(A^{*} A\right)-\Phi_{0}\left(A A^{*}\right) \text {. }
$$

Really,

$$
\mathrm{Sp} \exp \left(-t A^{*} A\right)-\mathrm{Sp}\left(-t A A^{*}\right)=\operatorname{index} A
$$

because non-zero eigenvalues of $A^{*} A$ and $A A^{*}$ coincide and their contributions in (4) cancel. Taking the limit $t \rightarrow 0$ we obtain (3) from (4). Combining (3) and the Seeley formula one can get the integral formula for index. The elliptic operators on compact manifolds can be considered as bounded operators in Sobolev spaces too. The Sobolev space $W_{p}^{k}(\xi)$ can be defined as a completion of $\Gamma(\xi)$ with respect to the norm

$$
\|f\|_{(p)}^{(k)}=\left\|B^{k / 2} f\right\|_{(p)},
$$

where $B$ is a positive second order elliptic operator, $\|g\|_{(p)}$ denotes the $L^{p}$-norm. [The space $W_{p}^{k}(\xi)$ and the topology in this space do not depend on operator $B$ and on the choice of hermitian structure in $\xi$.] One can prove that

$$
\|f\|_{(p)}^{(k)} \leqq \text { const }\|f\|_{\left(p^{\prime}\right)}^{\left(k^{\prime}\right)}
$$

if $p \geqq p^{\prime}, \frac{n}{p}-k \geqq \frac{n}{p^{\prime}}-k^{\prime}$. It follows from (5) that the space $W_{p^{\prime}}^{k^{\prime}}$ is imbedded in the space $W_{p}^{k}$. (This assertion is known as Sobolev's imbedding theorem.) Let $A$ be a differential operator of order $N$ acting from $\Gamma\left(\xi_{1}\right)$ into $\Gamma\left(\xi_{2}\right)$. The extension of this operator gives a bounded operator $A^{(k, p)}$ acting from $W_{p}^{k}\left(\xi_{1}\right)$ into $W_{p}^{k-N}\left(\xi_{2}\right)$. If $A$ is an elliptic operator then $l(A)=l\left(A^{(k, p)}\right)$. (Every solution of elliptic equation $A^{(k, p)} f=0$ is smooth and therefore can be considered as a solution of equation $A f=0$.) Analogously $l\left(A^{*}\right)=l\left(A^{(k, p) *}\right)$. In particular if $l\left(A^{*}\right)=0$ the operator $A^{(k, p)}$ maps $W_{p}^{k}\left(\xi_{1}\right)$ onto $W_{p}^{k-N}\left(\xi_{2}\right)$. 
At last we give the definition of elliptic complex. (This notion will not be used in present paper.) The sequence

$$
0 \longrightarrow \Gamma_{0} \stackrel{d_{0}}{\longrightarrow} \Gamma_{1} \stackrel{d_{1}}{\longrightarrow} \ldots \stackrel{d_{N-1}}{\longrightarrow} \Gamma_{N} \longrightarrow 0
$$

of linear spaces $\Gamma_{0}, \ldots, \Gamma_{N}$ and linear maps $d_{i}$ acting from $\Gamma_{i}$ into $\Gamma_{i+1}$ will be called a complex if

$$
d_{i+1} d_{i}=0
$$

It follows from (7) that the image of $d_{i-1}$ contains in the kernel of $d_{i}$ and therefore we can consider the coset space $H_{i}=\operatorname{Ker} d_{i} / \operatorname{Im} d_{i-1}$. The dimension of this space (the $i$-th Betti number) will be denoted by $h_{i}$. If $h_{i}=0$ for $i=0,1, \ldots, N$ we say that the complex is acyclic. The Euler characteric of the complex is defined as $\sum(-1)^{i} h_{i}$.

If $\Gamma_{i}=\Gamma\left(\eta_{i}\right)$, where $\eta_{i}$ is a vector bundle provided with hermitian structure, and the operators

$$
\Delta_{i}=d_{i}^{*} d_{i}+d_{i-1} d_{i-1}^{*}
$$

are elliptic we will say that the complex is elliptic. It is easy to check that

$$
\begin{aligned}
d_{i} \Delta_{i} & =\Delta_{i+1} d_{i}, \\
d_{i-1}^{*} \Delta_{i} & =\Delta_{i-1} d_{i-1}^{*} \\
\operatorname{Ker} \Delta_{i} & =\left(\operatorname{Ker} d_{i}\right) \cap\left(\operatorname{Ker} d_{i-1}^{*}\right) .
\end{aligned}
$$

One can prove that in this case $\Gamma_{i}$ is a direct sum of $\operatorname{Im} d_{i-1}, \operatorname{Im} d_{i}^{*}$ and $\operatorname{Ker} \Delta_{i}$ (Hodge decomposition). In other words each element $a \in \Gamma_{i}$ admits unique representation in the form $x+y+z$ where $x=d_{i-1} u, y=d_{i}^{*} v, d_{i} z=0, d_{i-1}^{*} z=0$.

It follows from Hodge decomposition that $\operatorname{Ker} d_{i}=\operatorname{Im} d_{i-1}+\operatorname{Ker} \Delta_{i}$ and therefore $h_{i}=l\left(\Delta_{i}\right)$.

The Euler characteristic of elliptic complex can be interpreted as index of elliptic operator. Namely, we can define the operator $\mathrm{T}$ acting from space $\Gamma_{\text {even }}=\Gamma_{0} \dot{+} \Gamma_{2} \dot{+} \ldots$ into the space $\Gamma_{\text {odd }}=\Gamma_{1} \dot{+} \Gamma_{3} \dot{+} \ldots$ as an operator transforming the sequence $\left(a_{0}, a_{2}, \ldots\right) \in \Gamma_{\text {even }}$ into the sequence $\left(b_{1}, b_{3}, \ldots\right) \in \Gamma_{\text {odd }}$, where $b_{2 i-1}=d_{2 i-1}^{*} a_{2 i}$ $+d_{2 i-2} a_{2 i-2}$. It is easy to prove that $T$ is an elliptic operator and index $T=\sum^{2}(-1)^{i} h_{i}$.

Let $\Gamma^{k}$ be the space of $k$-forms on the manifold $M$. If $d_{k}=d$ is the exterior differential, the spaces $\Gamma^{k}$ and maps $d_{k}$ form a complex (de Rham complex of the manifold $M$ ). The Betti numbers of this complex are equal to the Betti numbers of the manifold $M$. If $M$ is a compact riemannian manifold then de Rham complex can be considered as an elliptic complex. An obvious generalization of de Rham complex can be obtained, if we denote by $\Gamma^{k}$ the space of $k$-forms taking values in the fibres of an $O(m)$-bundle and $d_{k}$ denotes the exterior differential with respect to the gauge field with vanishing strength $(F=0)^{3}$.

3 If one can find such a field the bundle is called flat. There exists a natural correspondence between flat bundles and representations of the group $\pi_{1}(M)$ into $O(m)$ 


\section{Section 4. Instantons}

Let us consider a principal fibration $\xi(E, M, G, p)$ where $M$ is four-dimensional compact oriented riemannian manifold, $G$ is a simple compact Lie group. The euclidean action of the gauge field $A$ in $\xi$ can be defined by formula

$$
S(A)=\frac{1}{2 g^{2}}(F, F)=\frac{1}{2 g^{2}} \int\left\langle F_{\alpha \beta}, F^{\alpha \beta}\right\rangle d V,
$$

where $F$ is the strength of the field $A$. [As we have explained in Sect. $2 F$ can be considered as 2-form on $M$ taking values in the fibres of the associated vector bundle $\xi_{\tau}\left(E_{\tau}, M, \mathscr{G}, p_{\tau}\right)$ where $\mathscr{G}$ is the Lie algebra of $G$ and $G$ acts on $\mathscr{G}$ by means of adjoint representation. The scalar product $(F, F)$ is defined by means of invariant scalar product in $\mathscr{G}$ and riemannian metric in $M$.]

It is well known that $(F, * F)$ does not depend on the field $A$; this number is determined completely by topological type of the fibration $\xi$. By appropriate normalization of invariant scalar product in $\mathscr{G}$ the number

$$
q=\frac{1}{16 \pi^{2}}(F, * F)
$$

is an integer (see for example [35] or [1]). If $G$ is an abelian group, then $q=0$. We assume further that $G$ is a simple compact non-abelian group. Using that $(* F, * F)=(F, F)$ and $(F, * F)=(* F, F)$ we obtain that

$$
(F-* F, F-* F)=2(F, F)-2(F, * F) .
$$

It follows from obvious inequality $(F-* F, F-* F) \geqq 0$ that $(F, F) \geqq(F, * F)$ and $(F, F)=(F, * F)$ if and only if $F=* F$. We see that $S(A) \geqq 8 \pi^{2} g^{-2} q$ and $S(A)=8 \pi^{2} g^{-2} q$ if and only if the gauge field $A$ satisfies the duality equation

$$
F=* F \text {. }
$$

Without loss of generality we can suppose that $q \geqq 0$ because the sign of $q$ depends on the choice of orientation of $M$. [If $q<0$ then $S(A) \geqq 8 \pi^{2} g^{-2}|q|$ and $S(A)=8 \pi^{2} g^{-2}|q|$ if and only if $F=-* F$.]

We consider here the instantons on compact manifolds. However it is essential to note that the duality equation is conformally invariant and therefore each instanton on the sphere $S^{4}$ with usual metric can be considered as an instanton on the euclidean space.

Let $A$ be a gauge field satisfying the duality Eq. (3). We will study the solutions $A^{\prime}$ of the duality equation in the neighbourhood of $A$ imposing the gauge condition

$$
d_{A}^{*}\left(A^{\prime}-A\right)=0 .
$$

We consider for definiteness the case $G=\mathrm{SU}(2), M$ is a sphere $S^{4}$ with usual metric.

Theorem 1. There exists (8q-3)-parameter family of solutions of duality Eq. (3) satisfying the condition (4). 
To prove this theorem we search the solutions of (3), (4) in the form $A^{\prime}=A+a$ where $a$ can be considered as a 1 -form on $M$ taking values in the fibres of the bundle $\xi_{\tau}\left(E_{\tau}, M, \mathscr{G}, p_{\tau}\right)$. If $A^{\prime}$ satisfies (3) then $a$ must satisfy

$$
P\left(d_{A} a+[a, a]\right)=0
$$

(here $P=\frac{1}{2}(1-*)$ and $\left.[a, a]\right)$, where $a=a_{\lambda} d x^{\lambda}$ is defined as a 2 -form $\left[a_{\lambda}, a_{\mu}\right] d x^{\lambda} \wedge d x^{\mu}$. The gauge condition (4) takes the form

$$
d_{A}^{*} a=0 .
$$

Let us consider at first the Eqs. (5), (6) neglecting the non-linear terms in (5). In this approximation the Eqs. (5), (6) can be written in the form $T a=0$ where $T$ is a linear operator transforming 1-form $a$ into a pair $(f, g)$ where $f=P d_{A} a$ is an antidual 2-form $(f=-* f)$ and $g$ is a 0 -form $d_{A}^{*} a$ (all forms under consideration take values in the fibres of $\xi_{\tau}$ ). The space of 1 -forms will be denoted by $\Gamma_{1}$, the space of 0 -forms - by $\Gamma_{0}$ and the space of antidual 2-forms by $\Gamma_{2}$. One can construct bundles $\eta_{1}$ and $\eta_{2}$ in such a way that $\Gamma_{1}$ can be considered as $\Gamma\left(\eta_{1}\right)$ and space $\Gamma_{2}+\Gamma_{0}$ consisting of pairs $(f, g)$ where $f$ is antidual 2-form, $g$ is 0 -form can be considered as $\Gamma\left(\eta_{2}\right)$. It is easy to check that $T$ is an elliptic operator acting from $\Gamma_{1}$ into $\Gamma_{2}+\Gamma_{0}$. The riemannian metric in $M$ and invariant scalar product in $\mathscr{G}$ induce scalar products in $\Gamma_{1}$ and $\Gamma_{2} \dot{+} \Gamma_{0}$ so that we can consider the adjoint operator $T^{*}$ acting from $\Gamma_{2}+\Gamma_{0}$ in $\Gamma_{1}$. The operator $T^{*}$ transforms the pair $(f, g) \in \Gamma_{2}+\Gamma_{0}$ into the form $d_{A}^{*} f+d_{A} g \in \Gamma_{1}$.

Lemma 1. The equation $T^{*}(f, g)=0$ has only zero solution [i.e. $l\left(T^{*}\right)=0$ ].

Lemma 2. The index of the operator $T$ is equal to $8 q-3$.

The proof of Lemma 1 will be given at the end of this section. The methods of calculation of index will be discussed in the Appendix I.

It follows from the lemmas that $l(T)=$ index $T+l\left(T^{*}\right)=8 q-3$. We see that in linear approximation the Eqs. (5), (6) have $(8 q-3)$-parameter family of solutions. To obtain rigorously the existence of $(8 q-3)$-parameter solution of $(5),(6)$ we consider the non-linear operator $R$ which transforms 1 -form $a \in \Gamma_{1}$ into the pair $(f, g) \in \Gamma_{2}+\Gamma_{0}$ where

$$
f=P\left(d_{A} a+[a, a]\right), \quad g=d_{A}^{*} a .
$$

It is convenient to regard $R$ as an operator acting in Sobolev spaces. Namely $R$ can be extended on $W_{p}^{k}\left(\eta_{1}\right)$ if $p>4$ and the extended operator $\tilde{R}$ acts from $W_{p}^{k}\left(\eta_{1}\right)$ into $W_{p}^{k-1}\left(\eta_{2}\right)$. [This assertion can be deduced from Sobolev imbedding theorem. Really it is easy to check that the non-linear part of $R$ can be considered as an operator acting from $W_{p}^{k}\left(\eta_{1}\right)$ into $W_{p / 2}^{k}\left(\eta_{2}\right)$ and for $p>4$ the space $W_{p / 2}^{k}$ is imbedded in $\left.W_{p}^{k-1}\right]$. Moreover the operator $\tilde{R}$ is continuously differentiable (i.e. $\tilde{R}$ is a $C^{1}$-map); the differential of $R$ at the point $a=0$ is the linear operator $\tilde{T}$ obtained by means of extension of $T$ on $W_{p}^{k}\left(\eta_{1}\right)$. It follows from Lemma 1 that the operator $\tilde{T}$ maps $W_{p}^{k}\left(\eta_{1}\right)$ onto $W_{p}^{k-1}\left(\eta_{2}\right)$. We can apply therefore the infinite-dimensional version of implicit function theorem (see for instance [33]) and obtain that the equation $\tilde{R} a=0$ has a family of solutions depending on $l(\tilde{T})$ parameters. The solutions of elliptic equation $\tilde{R} a=0$ are smooth and hence can be considered as 
solutions of equation $R a=0$. To complete the proof of Theorem 1 we note that $l(\tilde{T})=l(T)$ and use Lemma 2.

Now we must give the proof of Lemma 1. This proof is based on the following assertion.

Lemma 3. If $\psi$ is a non-zero field transforming according irreducible representation $V$ of SU(2) (i.e. $\psi$ is a section of vector bundle $\xi_{V}$ associated with principal fibration $\xi)$ and there exists a gauge field $A$ in $\xi$ satisfying $d_{A} \psi=0$ then the topological number q vanishes.

Really as we have mentioned in Sect. 2 the holonomy group of the field $A$ is abelian if the conditions of the Lemma 3 are fulfilled. Therefore the field $A$ is gauge equivalent to a gauge field taking values in the abelian Lie algebra and hence $q=0$.

To prove that $l\left(T^{*}\right)=0$ in the case $M=S^{4}$ we note that $l\left(T^{*}\right)=l\left(T T^{*}\right)$ and consider the equation

$$
T T^{*}(f, g)=0,
$$

where $f \in \Gamma_{2}, g \in \Gamma_{0}$. The operator $T T^{*}$ transforms the pair $(f, g) \in \Gamma_{2}+\Gamma_{0}$ into the pair $\left(\Delta_{2}^{A} f, \Delta_{0}^{A} g\right)$ where the operator $\Delta_{2}^{A}$ acting in $\Gamma_{2}$ and the operator $\Delta_{0}^{A}$ acting in $\Gamma_{0}$ are defined by the formulae

$$
\begin{aligned}
& \Delta_{2}^{A}=P d_{A} d_{A}^{*}, \\
& \Delta_{0}^{A}=d_{A}^{*} d_{A} .
\end{aligned}
$$

The Eq. (7) breaks up into equations

$$
\begin{aligned}
& \Delta_{2}^{A} f=0, \\
& \Delta_{0}^{A} g=0 .
\end{aligned}
$$

It follows from (8) that

$$
\left(d_{A} f, d_{A} f\right)=\left(d_{A}^{*} d_{A} f, f\right)=\left(* d_{A}^{*} d_{A} f, * f\right)=\left(d_{A} d_{A}^{*} f, f\right)=\left(\Delta_{2}^{A} f, f\right)=0
$$

and therefore $d_{A} f=0$. Now we obtain from (9) that $\left(d_{A} g, d_{A} g\right)=\left(\Delta_{0}^{A} g, g\right)=0$ and hence $d_{A} g=0$. Using Lemma 3 we conclude that $g=0$ and hence $l\left(\Delta_{0}^{A}\right)=0$. To prove that $l\left(\Delta_{2}^{A}\right)=0$ we consider the form $\tilde{f}$ corresponding to $f$ into euclidean space (one can say that $\tilde{f}$ is the form $f$ in stereographic coordinates). The form $f$ is smooth therefore the form $\tilde{f}$ has the asymptotics

$$
\tilde{f} \approx \frac{C_{\mu v}}{|x|^{4}}\left(\delta_{\mu \varrho}-\frac{x_{\mu} x_{\varrho}}{|x|^{2}}\right)\left(\delta_{v \sigma}-\frac{x_{v} x_{\sigma}}{|x|^{2}}\right) d x_{\varrho} \wedge d x_{\sigma}
$$

for $|x| \rightarrow \infty$. (To check this assertion one can note that the map $x_{\mu} \rightarrow|x|^{-2} x_{\mu}$ transforms $\tilde{f}$ into a smooth form.) The equations $d_{A} f=0, f=-* f$ are conformally invariant and therefore

$$
\begin{aligned}
& d_{A}^{*} d_{A} \tilde{f}=0, \\
& \tilde{f}=-* \tilde{f} .
\end{aligned}
$$


In euclidean space the Eqs. (11), (12) breaks up into independent equations of the form

$$
\nabla_{\mu}^{A} \nabla_{\mu}^{A} u=0
$$

for coefficient functions of $\tilde{f}$. (This fact can be verified by direct calculation. It follows also from assertions of Sect. 5.) Using the asymptotics (10) of $\tilde{f}$ we obtain that $\left(\nabla_{\mu}^{A} u, \nabla_{\mu}^{A}\right)=-\left(\nabla_{\mu}^{A} \nabla_{\mu}^{A} u, u\right)=0$ and therefore $\nabla_{\mu}^{A} u=0$.

From Lemma 3 we conclude that $u=0$ and hence $f=0$, i.e. $l\left(\Delta_{2}^{A}\right)=0$.

The operators $\Delta_{2}^{A}, \Delta_{0}^{A}$ introduced above will be important in the Sect. 6 as well as the operator

$$
\Delta_{1}^{A}=T^{*} T=d_{A}^{*} P d_{A}+d_{A} d_{A}^{*}
$$

acting in $\Gamma_{1}$. We use the notations $h_{i}^{A}=l\left(\Delta_{i}^{A}\right)$ for $i=0,1,2$. Noting that $l\left(\Delta_{1}^{A}\right)=l(T)$ we see that $h_{1}^{A}=8 q-3$ in the case under consideration.

We have assumed that $M$ is a sphere with the usual metric. However it is well known that the number $l\left(T^{*}\right)$ cannot increase by small variation of operator $T^{*}$ and therefore $l\left(T^{*}\right)=0$ if the metric on $M$ is sufficiently near to the usual one. We see that the assertion of Theorem 1 remains correct by small variation of usual metric of the sphere.

We found that the solutions of (3) in a neighbourhood of $A$ satisfying (4) form $(8 q-3)$-dimensional manifold. It is important to note that the gauge condition (4) eliminate the gauge freedom. Really, let us consider the infinitesimal gauge transformation determined by $\omega \in \Gamma_{0}$. If $A^{\prime}$ satisfies (3), (4) the gauge equivalent field $A^{\prime}+d_{A^{\prime}} \omega$ cannot satisfy (4) because the equation $d_{A}^{*} d_{A^{\prime}} \omega$ has only zero solution if $A^{\prime}$ is sufficiently near to $A$ [the operator $d_{A}^{*} d_{A^{\prime}}$ is obtained from $\Delta_{0}^{A}=d_{A}^{*} d_{A}$ by means of small variation and $l\left(\Delta_{0}^{A}\right)=0$ as it follows from Lemma 1]. One can replace here infinitesimal gauge transformation by small gauge transformations.

Another proof of theorem using the elliptic complex

$$
0 \longrightarrow \Gamma_{0} \stackrel{d_{A}}{\longrightarrow} \Gamma_{1} \stackrel{P d_{A}}{\longrightarrow} \Gamma_{2} \longrightarrow 0
$$

is given in [11]. This complex is equivalent to the elliptic operator $T$.

The main part of considerations above can be applied also in the case of arbitrary simple non-abelian compact Lie group $G$. Generalizing the considerations above one can prove that $h_{0}^{A}$ is equal to the dimension of the largest subgroup of $G$ which commutes with holonomy group of $A[11,15]$. If $h_{0}^{A}=0$ then in the case $M=S^{4}$ our arguments show that $h_{2}^{A}=0$ and the number 0 . instanton parameters is equal to index $T$. This index can be calculated easily (see Appendix I). There exists also another proof of equality $h_{2}^{A}=0$ which shows that this equality is valid for arbitrary instanton on the sphere $S^{4}[11,15]$.

\section{Section 5. Fermions in the Field of Instanton}

Let us consider now the euclidean Dirac operator $D$ in the gauge field $A$ where $A$ takes values in the Lie algebra of the compact simple Lie group $G$ and $D$ acts on the byspinor $\psi$. We suppose that $\psi$ has $N$ isotopic indices and transforms according to a representation of the group $G$ which will be denoted by $V$. The fields under consideration are defined on four-dimensional riemannian manifold $M$. We 
denote by $B$ the operator $D$ considered only on the right spinors. The operator $B$ transforms the right spinors into the left spinors and the operator $B^{*}$ coincides with the operator $D$ on the left spinors. We will study the zero modes of the operator $D$ (i.e. the solutions of equation $D \psi=0$ ). It is evident that $l(B)$ is a number of right zero modes of $D$ and $l\left(B^{*}\right)$ is a number of left zero modes of $D$. By definition

$$
\text { index } B=l(B)-l\left(B^{*}\right) \text {. }
$$

This index is well known [31-33]. (The methods of calculation of this index are discussed in Appendix I.) We obtain

Theorem 2. The difference between the number of right zero modes and the number of left zero modes of Dirac operator on the compact riemannian manifold $M$ is equal to

$$
\alpha_{V} q+\frac{N}{8} \tau(M)
$$

where $q$ is the topological number of the gauge field, $\tau(M)$ is the signature of the manifold $M$ and $\alpha_{V}$ is the Dynkin index of the representation $V$.

The signature of riemannian manifold can be expressed through the Riemann tensor $R_{\alpha \beta \gamma \delta}$ by the formula

$$
\tau(M)=\frac{1}{96 \pi^{2}} \int \varepsilon^{\mu \nu \alpha \beta} R_{\mu \nu \lambda \varrho} R_{\alpha \beta}^{\lambda \varrho} d V .
$$

The Dynkin index of the representation is equal to

$$
\alpha_{V}=\frac{\langle v(x), v(y)\rangle}{\langle x, y\rangle}
$$

where $v$ denotes the homomorphism of the Lie algebra $\mathscr{G}$ of $G$ into the Lie algebra of SU(n), $x, y \in \mathscr{G}$ and the scalar product in the Lie algebras is normalized by the condition that the maximal length of the root is equal to 2. The Dynkin index coincides up to trivial factor with the quadratic Casimir operator of the representation $V$. [The signature and the Dynkin index admit simple homological interpretations. For example $\alpha_{V}$ can be defined by formula

$$
V_{*} a=\alpha_{V} b,
$$

where $a$ and $b$ are the generators of the homology groups $H_{3}(G, Z)$ and $H_{3}(\mathrm{SU}(n), Z)$ and $V_{*}$ denotes the homomorphism of homology groups induces by $V$. It follows from (4) that $\alpha_{V}$ is an integer.]

The well known formula (1) for index of Dirac operator was rediscovered by Coleman (unpublished) and Kiskis [12] in the flat case by means of Adler-BellJackiv anonaly. As was mentioned in [8] the Adler-Bell-Jackiv anomaly can be obtained from local index theorem and therefore the proof based on the index theorem and the proof using Adler-Bell-Jackiv anomaly are closely related.

The signature of the sphere $S^{4}$ is equal to zero. The Dynkin index of the $l$-dimensional irreducible representation of the group $S U(2)$ is equal to 
$\frac{1}{6}(l-1) l(l+1)$. Therefore in the case $M=S^{4}, G=\mathrm{SU}(2)$ the index $B$ is equal to $q$ if $V$ is the two-dimensional (spinor) representation of $S U(2)$ and to $4 q$ if $V$ is the adjoint representation of SU(2). Let us consider now the case when the manifold $M$ is flat (for example $M$ is an euclidean space or $M$ is a torus).

Theorem 3. If $M$ is a torus then the Dirac equation in the field of instanton cannot by satisfied by non-zero left spinor [i.e. $l\left(B^{*}\right)=0$ ]. form

The operator $D$ on a torus can be written as in euclidean space in the usual

$$
D=\gamma^{\mu} \nabla_{\mu}=\gamma^{\mu}\left(\frac{\partial}{\partial x^{\mu}}+v\left(A_{\mu}\right)\right)
$$

Hence

$$
\begin{aligned}
D^{2} & =\gamma^{\mu} \gamma^{v} \nabla_{\mu} \nabla_{v}=\frac{1}{2} \gamma^{\mu} \gamma^{v}\left(\nabla_{\mu} \nabla_{v}+\nabla_{v} \nabla_{\mu}\right)+\frac{1}{2} \gamma^{\mu} \gamma^{v}\left(\nabla_{\mu} \nabla_{v}-\nabla_{v} \nabla_{\mu}\right) \\
& =-\nabla_{\mu} \nabla_{\mu}+\frac{1}{2} \gamma^{\mu} \gamma^{v} v\left(F_{\mu \nu}\right) .
\end{aligned}
$$

Using that

$$
\gamma^{\mu} \gamma^{\nu} \gamma^{5}=-\frac{1}{2} \varepsilon^{\mu \nu \varrho \sigma} \gamma^{\varrho} \gamma^{\sigma}
$$

we obtain that the operator $D^{2}$ on the left spinors (i.e. the operator $B B^{*}$ ) is equal to $-\nabla_{\mu} \nabla_{\mu}$. If $\psi$ is a left spinor satisfying $D \psi=0$ then $\left(\nabla_{\mu} \psi, \nabla_{\mu} \psi\right)=-\left(\nabla_{\mu} \nabla_{\mu} \psi, \psi\right)$ $=\left(D^{2} \psi, \psi\right)=0$ therefore $\nabla_{\mu} \psi=0$. If follows now from Lemma 3 that $\psi=0$.

The proof above shows that the assertion of Theorem 3 remains correct in the case when $M$ is an euclidean space. However in this case we must consider only solutions decreasing fast enough to justify the equality $\left(\nabla_{\mu} \psi, \nabla_{\mu} \psi\right)$ $=-\left(\nabla_{\mu} \nabla_{\mu} \psi, \psi\right)=0$.

There exists a remarkable connection between linearized duality equation in euclidean space and Dirac equation. It is easy to check that

$$
T\left(\bar{\sigma} \gamma^{\mu} \varphi(x)\right)=\left(\frac{1}{2} \bar{\sigma}\left(\gamma^{\mu} \gamma^{v}-\gamma^{v} \gamma^{\mu}\right) B \varphi(x),-\bar{\sigma} B \varphi(x)\right)
$$

where spinor $\varphi(x)$ transform according adjoint representation of the group $G, \sigma$ is a fixed right spinor and $\varphi(x)$ is a right spinor depending on $x$. It follows from (5) that

$$
\begin{aligned}
T^{*}\left(\frac{1}{2} \bar{\sigma}\left(\gamma^{\mu} \gamma^{v}-\gamma^{v} \gamma^{\mu}\right) \psi, \bar{\sigma} \psi\right) & =\bar{\sigma} \gamma^{\mu} B^{*} \psi \\
T T^{*}\left(\frac{1}{2} \bar{\sigma}\left(\gamma^{\mu} \gamma^{\nu}-\gamma^{v} \gamma^{\mu}\right) \psi, \bar{\sigma} \psi\right) & =\left(\frac{1}{2} \bar{\sigma}\left(\gamma^{\mu} \gamma^{\nu}-\gamma^{v} \gamma^{\mu}\right) B B^{*} \psi,-\bar{\sigma} B B^{*} \psi\right)
\end{aligned}
$$

[here $\psi(x)$ is a left spinor, the antisymmetric tensor $\bar{\sigma}\left(\gamma^{\mu} \gamma^{\nu}-\gamma^{\nu} \gamma^{\mu}\right) \psi(x)$ determines antidual 2-form.]

We know that the operator $D^{2}=B B^{*}$ on left spinors is equal to $-\nabla_{\mu} \nabla_{\mu}$. Using this assertion and (6) we obtain that the equation $T T^{*}(f, g)=0$ breaks up into four independent equations of the form $\nabla_{\mu} \nabla_{\mu} u=0$ (this fact was used by the proof of Lemma 1). 


\section{Section 6. Determinants of Elliptic Operators}

Let $A$ be a non-negative self-adjoint elliptic operator on compact manifold. The determinant of this operator in usual sense is infinite. We say that the expression

$$
\operatorname{det}_{\varepsilon} A=\exp \left(-\sum_{i} \int_{\varepsilon}^{\infty} t^{-1} \exp \left(-\lambda_{i} t\right) d t\right)
$$

is obtained from the (infinite) determinant of $A$ by means of proper time cutoff (here $\lambda_{i}$ run over positive eigenvalues of $\left.A\right)^{4}$. The expression (1) for $\operatorname{det}_{\varepsilon} A$ can be rewritten in the form

$$
\log \operatorname{det}_{\varepsilon} A=-\int_{\varepsilon}^{\infty} t^{-1} \operatorname{Sp}(\exp (-t A)-\Pi(A)) d t .
$$

Here and later $\Pi(A)$ denotes the projector on the kernel of $A$ [i.e. $\Pi(A) f=\sum\left(f, \varphi_{i}\right) \varphi_{i}$ where $\varphi_{i}$ run over zero modes of $\left.A\right]$. The asymptotics of $\log \operatorname{det}_{\varepsilon} A$ for $\varepsilon \rightarrow 0$ can be obtained by means of asymptotics of $\operatorname{Sp} \exp (-t A)$ for $t \rightarrow 0$. It is well known [34] that for $t \rightarrow 0$

$$
\mathrm{Sp} \exp (-t A) \approx \sum \alpha_{k} t^{-k}
$$

[in notations of Sect. $3 \alpha_{k}=\Phi_{k}(A)$ ]. Using (2) and (3) we see that for $\varepsilon \rightarrow 0$ the divergent part of $\log \operatorname{det}_{\varepsilon} A$ can be represented in the form

$$
-\sum_{k>0} k^{-1} \alpha_{k} \varepsilon^{-k}+\left(\alpha_{0}-p\right) \log \varepsilon
$$

where $\alpha_{k}=\Phi_{k}(A)$ and $p$ denotes the number of zero modes of $A$. The finite part of

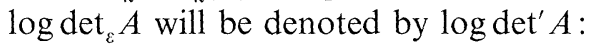

$$
\log \operatorname{det}^{\prime} A=\lim _{\varepsilon \rightarrow 0}\left(\log \operatorname{det}_{\varepsilon} A+\sum_{k>0} k^{-1} \alpha_{k} \varepsilon^{-k}-\left(\alpha_{0}-p\right) \log \varepsilon\right) .
$$

It is easy to check that $\log \operatorname{det}^{\prime} A$ can be represented in the form

$$
\begin{aligned}
\log \operatorname{det}^{\prime} A= & -\int_{0}^{\infty} t^{-1}\left(\operatorname{Sp} \exp (-t A)-\sum_{k>0} \alpha_{k} t^{-k}\right. \\
& \left.-\theta(1-t) \alpha_{0}-\theta(t-1) p\right) d t .
\end{aligned}
$$

In present paper we will define the regularized determinant of non-negative elliptic operator by formula (4). There exists another definition of regularized determinant based on the notion of zeta function (see [25] for instance). All our results remain correct by this definition of regularized determinant too.

The zeta function $\zeta(s \mid A)$ of non-negative elliptic operator $A$ for large $\operatorname{Re} s$ can be defined by formula

$$
\zeta(s \mid A)=\sum \lambda_{i}^{-s}=\frac{1}{\Gamma(s)} \int_{0}^{\infty} t^{s-1} \operatorname{Sp}(\exp (-t A)-\Pi(A)) d t,
$$

4 The definition of $\operatorname{det}_{\varepsilon} A$ is prompted by the formula

$$
\log \operatorname{det} A-\log \operatorname{det} A_{0}=-\int_{0}^{\infty} t^{-1}\left(\operatorname{Sp} \exp (-t A)-\operatorname{Sp} \exp \left(-t A_{0}\right)\right) d t
$$

which is valid for finite-dimensional operators 
where $\lambda_{i}$ run over positive eigenvalues of $A$. For other $s$ zeta function must be defined by means of analytic continuation. For $t \rightarrow \infty$ the integrand in (6) decreases exponentially, the asymptotics of the integrand for $t \rightarrow 0$ is given by Seeley formula. Using these facts one can prove that $\zeta(s \mid A)$ is meromorphic function; if the asymptotics of $\operatorname{Sp} \exp (-t A)$ for $t \rightarrow 0$ contains a term $\alpha_{k} t^{-k}, k \neq 0$ then the function $\Gamma(s) \zeta(s \mid A)$ has a simple pole at the point $s=k$ with residue $\alpha_{k}$. For $s \rightarrow 0$ we have $s \Gamma(s) \rightarrow 1$ and therefore $\zeta(s \mid A)$ is analytic at the point $s=0$ and $\zeta(0 \mid A)=\alpha_{0}-p$. The regularized determinant $D(A)$ can be defined now by the formula

$$
\log D(A)=-\left.\frac{d}{d s} \zeta(s \mid A)\right|_{s=0} .
$$

[This definition is correct because $\zeta(s \mid A)$ is analytic at $s=0$.]

The analytic extension of $\zeta(s \mid A)$ in the half-plane $\operatorname{Re} s \geqq 0$ can be written in the form

$$
\begin{aligned}
\zeta(s \mid A)= & \frac{1}{\Gamma(s)}\left(\sum_{k>0} \frac{\alpha_{k}}{s-k}+\frac{\alpha_{0}-p}{s}+\int_{1}^{\infty}(\operatorname{Sp} \exp (-t A)-p) t^{s-1} d t\right. \\
& \left.+\int_{0}^{1}\left(\operatorname{Sp} \exp (-t A)-\sum_{k \geqq 0} \alpha_{k} t^{-k}\right) t^{s-1} d t\right) .
\end{aligned}
$$

Using (7) and (8) we obtain

$$
\begin{aligned}
\log D(A)= & \sum_{k>0} k^{-1} \alpha_{k}+\Gamma^{\prime}(1)\left(\alpha_{0}-p\right) \\
& -\int_{1}^{\infty} t^{-1}(\operatorname{Sp} \exp (-t A)-p) d t \\
& -\int_{0}^{1} t^{-1}\left(\operatorname{Sp} \exp (-t A)-\sum_{k \geqq 0} \alpha_{k} t^{-k}\right) d t .
\end{aligned}
$$

Comparing (5) and (9) we conclude that

$$
\log \operatorname{det}^{\prime} A=\log D(A)-\Gamma^{\prime}(1)\left(\alpha_{0}-p\right) .
$$

The eigenvalues of $A^{K}$ are expressed through eigenvalues of $A$ as $\lambda_{i}^{K}$, therefore $\zeta\left(s \mid A^{K}\right)=\zeta(K s \mid A)$ and hence

$$
\log D\left(A^{K}\right)=K \log D(A) .
$$

From $\zeta(s \mid \varrho A)=\varrho^{s \zeta}(s \mid A)$ we conclude that

$$
\log D(\varrho A)=\log D(A)-\zeta(0 \mid A) \log \varrho .
$$

For arbitrary elliptic operator we define $D(A)$ by the formula

$$
\log D(A)=\frac{1}{2} \log D\left(A^{*} A\right)=-\left.\frac{1}{2} \frac{d}{d s} \zeta\left(s \mid A^{*} A\right)\right|_{s=0} .
$$

It follows from (11) that for non-negative self-adjoint operator $A$ this definition is equivalent to the definition by means of (7). 
The definitions of regularized determinants given above can be applied for some non-elliptic operators too. However the existence of determinants of nonelliptic operators cannot be guaranteed.

One can replace $\operatorname{det}^{\prime} A$ by $D(A)$ in considerations of present paper.

\section{Section 7. Instanton Contribution in Green Functions}

In present section we express the instanton contribution in euclidean Green functions through regularized determinants of elliptic operators. Our considerations in this section will be heuristic.

We consider the euclidean action

$$
S=\int \bar{\psi} D_{A} \psi d V-\int \bar{\psi} \mathscr{M} \psi d V+S_{Y M}
$$

describing fermions $\psi$ interacting with gauge fields $A_{\mu}$ [the gauge fields take values in the Lie algebra $\mathscr{G}$ of the simple non-abelian compact gauge group $G$; the fermions fields transform according the representation $T$ of $G, S_{Y M}$ is the YangMills euclidean action (4.1), $D_{A}$ is the euclidean Dirac operator in the gauge field, the eigenvalues of the matrix $M$ determine the masses of fermion fields].

The euclidean Green functions (Schwinger functions) can be defined formally as quotient of two functional integrals. Namely if $\Phi(A)$ is a gauge invariant functional we define the euclidean Green function by the formula

$$
I_{\Phi}=\frac{\int \Phi(A) \exp (-S) d A d \bar{\psi} d \psi}{\int \exp (-S) d A d \bar{\psi} d \psi}
$$

(here $\bar{\psi}, \psi$ must be considered as anticommuting variables [36]). For example we can take

$$
\Phi(A)=\varepsilon\left(x_{1}\right) \ldots \varepsilon\left(x_{n}\right)
$$

where

$$
\varepsilon(x)=\left\langle F_{\alpha \beta}(x), F^{\alpha \beta}(x)\right\rangle .
$$

Of course (1) is not well defined expression; the rigorous definition of (1) must include cutoff and renormalization.

After integration over fermion variables we obtain

$$
I_{\Phi}=\frac{\int \Phi(A) \operatorname{det}\left(D_{A}-\mathscr{M}\right) \exp \left(-S_{Y M}\right) d A}{\int \operatorname{det}\left(D_{A}-\mathscr{M}\right) \exp \left(-S_{Y M}\right) d A} .
$$

One can use the method of steepest descent for calculation of (2) in weak coupling case. We study the instanton contribution in (2). [One can construct approximate extremals as superposition of distant instantons and anti-instantons. These extremals and other approximate extremals must be taken into account by calculation of (2) but we consider the contribution of instantons only]. The contribution of instantons having topological number $q$ contains a small factor $\exp \left(-8 \pi^{2} g^{-2} q\right)$ for $q>0$, however this contribution is essential by calculation of numerator of (2) (for example in the case when the long range correlations are 
studied). The contribution of instantons having non-zero topological number in the denominator of (2) will be neglected. The constant factors in our formulae will be omitted.

The method of steepest descent for integrals of invariant functions is studied in Appendix II for finite-dimensional integrals. We will use formally the lemma proved at the end of Appendix II for the infinite-dimensional integrals. (To be rigorous one must make the lattice cutoff, apply the lemma in the finitedimensional case and remove the cutoff.) The euclidean Yang-Mills action $S_{Y M}$ plays the role of the function $d^{-1} g(x)$ entering in lemma. The functional $\frac{1}{2}(F, F)$ plays the role of the function $g(x)$. To calculate the Hessian of this functional on the manifold of instantons we note that for gauge fields having the form $A_{\mu}+q_{\mu}$ where $A_{\mu}$ is an instanton one can represent $(F, F)$ in the form

$$
\begin{aligned}
(F, F) & =16 \pi^{2} q+\frac{1}{2}(F-* F, F-* F) \\
& =16 \pi^{2} q+2\left(P d_{A} a, P d_{A} a\right)+\ldots \\
& =16 \pi^{2} q+2\left(d_{A}^{*} P d_{A} a, a\right)+\ldots
\end{aligned}
$$

[here $P=\frac{1}{2}(1-*)$, the terms having third and forth degree with respect to $a$ are omitted]. We see that the Hessian of functional $\frac{1}{2}(F, F)$ can be considered as determinant of the operator $\Delta^{\prime}=d_{A}^{*} P d_{A}=\left(P d_{A}\right)^{*} P d_{A}$ acting in the space of 1 -forms. By calculation of determinant only non-zero eigenvalues of $\Delta^{\prime}$ must be taken into account; these eigenvalues coincide with non-zero eigenvalues of the operator $\Delta_{2}^{A}=P d_{A}\left(P d_{A}\right)^{*}=P d_{A} d_{A}^{*} P$ acting in the space of antidual 2-forms: $f=-* f$ [all forms under consideration take the values in the fibres of the fibration $\left.\xi_{\tau}\left(E_{\tau}, M, \mathscr{G}, p_{\tau}\right)\right]$.

The group of local gauge transformations $G_{\infty}$ plays the role of the group $G$ in the lemma of Appendix II. The operator $\Delta_{0}^{A}=d_{A}^{*} d_{A}$ acting on 0 -forms plays the role of the operator $T$ in lemma (this assertion follows from identity

$$
\left(\Delta_{0}^{A} \varphi, \varphi\right)=\left(d_{A} \varphi, d_{A} \varphi\right)
$$

because the infinitesimal gauge transformation transforms the field $A$ into $d_{A} \varphi$ ).

Using the lemma we represent the $q$-instanton contribution in the form

$$
I_{q}=g^{-\sigma} \exp \left(-\frac{8 \pi^{2} q}{g^{2}}\right) \int_{R_{q}} \Phi(A) \frac{\lambda(A)}{\lambda(0)} d \mu_{0},
$$

where

$$
\lambda(A)=\operatorname{det}\left(D_{A}-\mathscr{M}\right)\left(\operatorname{det} \Delta_{2}^{A}\right)^{-1 / 2}\left(\operatorname{det} \Delta_{0}^{A}\right)^{1 / 2}\left(\text { volume } H^{A}\right)^{-1}
$$

and $\lambda(0)$ is given by the same formula with $A=0$. The integration in (3) goes over the manifold $R_{q}$ obtained from the manifold $N_{q}$ of regular instantons ${ }^{5}$ having topological number $q$ by means of identification of gauge equivalent fields, $H^{A}$ denotes the group of gauge transformations leaving invariant the instanton $A$ and $d \mu_{0}$ denotes the measure on $R_{q}$ corresponding to the natural riemannian metric. [If

5 The instanton $A$ is called regular if all instantons in the neighbourhood of $A$ have equivalent holonomy groups [15]. We do not take into account the irregular instantons because the measure of the set of irregular instantons is equal to zero (this assertion can be derived from [19]) 
$A$ and $A+a$ are instantons, $a \rightarrow 0$, then the distance between the corresponding points of $R_{q}$ can be defined as $\left(a+d_{A} \varphi, a+d_{A} \varphi\right)^{1 / 2}$, where $\varphi$ satisfies $d_{A}^{*}\left(a+d_{A} \varphi\right)=0$.] In the notations of the end of Sect. $4 \operatorname{dim} R_{0}=h_{0}^{1}=0$ and $\operatorname{dim} R_{q}=h_{1}^{A}$ where $A$ is a regular instanton. Further, $\operatorname{dim} H^{A}=h_{0}^{A}$ and $\operatorname{dim} H^{0}=h_{0}^{0}=\operatorname{dim} G$. The number $\sigma$ is equal to $\operatorname{dim} N_{q}-\operatorname{dim} N_{0}$. The (infinite) dimension of $N_{q}$ can be represented as $\operatorname{dim} R_{q}+\left(\operatorname{dim} G_{\infty}-\operatorname{dim} H^{A}\right)=h_{1}^{A}-h_{0}^{A}$ $+\operatorname{dim} G_{\infty}$, where $A$ is a regular instanton with topological number $q$. In particular $\operatorname{dim} N_{0}=h_{1}^{0}-h_{0}^{0}+\operatorname{dim} G_{\infty}=\operatorname{dim} G_{\infty}-\operatorname{dim} G$. We see that $\sigma=\left(h_{1}^{A}-h_{0}^{A}\right)$ $-\left(h_{1}^{0}-h_{0}^{0}\right.$ ) (one can avoid the operations with infinite quantity $\operatorname{dim} G_{\infty}$ introducing the lattice cutoff).

The determinants in (4) diverge. To obtain finite answer we must perform cutoff and renormalization. To eliminate the infrared divergences we will consider all fields on compact riemannian manifold $M$. (In other words we make the volume cutoff. Further one can assume that $M$ is a sphere of radius 1 for definiteness.) The momentum cutoff is essentially equivalent to the proper time cutoff of determinants if $\varepsilon=C A^{-2}$. (Here $\varepsilon$ denotes the lower bound of proper time and $A$ is the maximal momentum.)

In the case when $M$ is a sphere of radius $R$ and the coordinates on the sphere are dimensionless the dimension arguments show that $C=K R^{-2}$ where $K$ is a dimensionless constant. We see that the contribution of determinants in the divergent part of (3) is equal to $\exp (\chi(A)-\chi(0)) \log \Lambda$ where

$$
\varkappa(A)=-\Phi_{0}\left(\left(D_{A}-\mathscr{M}\right)^{2}\right)+\left(\Phi_{0}\left(\Delta_{2}^{A}\right)-h_{2}^{A}\right)-\left(\Phi_{0}\left(\Delta_{0}^{A}\right)-h_{0}^{A}\right) .
$$

[The linear and quadratic divergent terms cancel. To check this assertion we note that the coefficients $\Phi_{i}\left(\left(D_{A}-\mathscr{M}\right)^{2}\right), \Phi_{i}\left(\Delta_{2}^{A}\right), \Phi_{i}\left(\Delta_{0}^{A}\right)$ can be expressed through the Riemann tensor $R_{\alpha \beta \gamma \delta}$, strength of the gauge field $F_{\alpha \beta}$ and their covariant derivatives. However simple dimensionality arguments show that the expression of these coefficients for $i=1,2$ cannot contain the gauge field, therefore

$$
\Phi_{i}\left(\left(D_{A}-\mathscr{M}\right)^{2}\right)=\Phi_{i}\left(\left(D_{0}-\mathscr{M}\right)^{2}\right), \Phi_{i}\left(\Delta_{2}^{A}\right)=\Phi_{i}\left(\Delta_{l}^{0}\right), \Phi_{i}\left(\Delta_{0}^{A}\right)=\Phi_{i}\left(\Delta_{0}^{0}\right)
$$

for $i=1,2]$. One can see that $d \mu_{0}$ and (volume $\left.H^{A}\right)^{-1}$ also contribute in divergent part of (3), namely the contribution of $d \mu_{0}$ is equal to

$$
\Lambda^{\operatorname{dim} R_{q}}=\exp \left(h_{1}^{A} \log \Lambda\right)
$$

and the contribution of (volume $\left.H^{A}\right)^{-1}$ is equal to

$$
\Lambda^{-2 \operatorname{dim} H^{A}}=\exp \left(-2 h_{0}^{A} \log \Lambda\right) .
$$

To verify this assertion one can use the lattice cutoff. This cutoff is essentially equivalent to the momentum cutoff if the maximal momentum $\Lambda$ is connected with the lattice spacing $a$ by the formula $\Lambda=a^{-1}$. (We suppose here for simplicity that $M$ is a torus.) We fix the direction of all lattice bonds and consider the gauge field on the lattice as a $G$-valued function $g_{\gamma}$ on the lattice bonds [37]. The correspondence with the continuous case is given by the formula

$$
g_{\gamma}=T \exp \left(-\int_{\gamma} A_{\mu} d x^{\mu}\right)
$$


The integration in the integral for Schwinger function in lattice case goes over all $g_{\gamma}$ (i.e. we integrate over the direct product $\prod_{\gamma} G$ of gauge group $G$ assigned to each bond of the lattice). The measure of integration corresponds to the riemannian metric

$$
d s^{2}=\sum_{\gamma}\left\langle g_{\gamma}^{-1} d g_{\gamma}, g_{\gamma}^{-1} d g_{\gamma}\right\rangle
$$

If $a \rightarrow 0$ and $g_{\gamma}$ is given by (8) then

$$
d s^{2} \approx a^{-2} \int\left\langle\delta A_{\mu}, \delta A^{\mu}\right\rangle d V=\Lambda^{2}(\delta A, \delta A)
$$

We see that after the cutoff the integration over the manifold $R_{q}$ contains an extra factor $\Lambda^{\operatorname{dim} R_{q}}$. The lattice counterpart of the group of local gauge transformations is the direct product $\prod_{\alpha} G$ of gauge groups assigned to each lattice point $\alpha$. Each local gauge transformation $g(x)$ generates an element $\{g(\alpha)\} \in \prod_{\alpha} G$ and in the limit $a \rightarrow 0$ the riemannian metric in $\prod_{\alpha} G$ is connected with the metric in the group of local gauge transformations by the formula

$$
\begin{aligned}
d s^{2} & =\sum_{\alpha}\left\langle g_{\alpha}^{-1} d g_{\alpha}, g_{\alpha}^{-1} d g_{\alpha}\right\rangle \\
& \approx a^{-4} \int\left\langle g^{-1}(x) \delta g(x), g^{-1}(x) \delta g(x)\right\rangle d V .
\end{aligned}
$$

We see that after the cutoff the (volume $\left.H^{A}\right)^{-1}$ contains an extra factor $\Lambda^{-2 \operatorname{dim} H^{A}}$. The divergent factors arising from determinants, $d \mu_{0}$ and (volume $\left.H^{A}\right)^{-1}$ give together a factor

$$
\exp (\varrho(A)-\varrho(0)) \ln \Lambda,
$$

where

$$
\varrho(A)=\varkappa(A)+h_{1}^{A}-2 h_{0}^{A}=\Phi_{0}\left(\Delta_{1}^{A}\right)-2 \Phi_{0}\left(\Delta_{0}^{A}\right)-\Phi_{0}\left(\left(D_{A}-\mathscr{M}\right)^{2}\right) .
$$

[We have used here the equality

$$
\Phi_{0}\left(\Delta_{1}^{A}\right)-\mathrm{h}_{1}^{A}=\left(\Phi_{0}\left(\Delta_{2}^{A}\right)-h_{2}^{A}\right)+\left(\Phi_{0}\left(\Delta_{0}^{A}\right)-h_{0}^{A}\right)
$$

which can be derived from the coincidence of non-zero eigenvalues of operators $T T^{*}$ and $T^{*} T$ where $T$ is the operator considered in Sect. 4, $T^{*} T=\Delta_{1}^{A}$, $T T^{*}=\Delta_{2}^{A} \dot{+} \Delta_{0}^{A}$.] The last expression for $\varrho(A)$ does not contain the numbers $h_{i}^{A}$. The numbers $\Phi_{0}\left(\left(D_{A}-\mathscr{M}\right)^{2}\right), \Phi_{0}\left(\Delta_{1}^{A}\right), \Phi_{0}\left(\Delta_{0}^{A}\right)$ can be calculated by Seeley formula (see Appendix I). We obtain from this calculation that the divergent factor (9) and the divergent factor coming from usual one-loop renormalization of coupling constant cancel. We use here the well-known formula [46]

$$
\frac{1}{g^{2}}=\frac{1}{g_{\text {phys }}^{2}}+\frac{1}{8 \pi^{2}}\left(\frac{11}{6} \alpha_{\tau}-\frac{2}{3} \alpha_{T}\right) \log \frac{\Lambda}{v}
$$


giving the expression of the bare coupling constant $g$ through the physical coupling constant $g_{\text {phys }}$ and the subtraction point $v$.

In this formula $\alpha_{\tau}$ denotes the Dynkin index of the adjoint representation of the group $G$ and $\alpha_{T}$ denotes the Dynkin index of the representation $T$.

We can write now the expression of instanton contribution through regularized determinants. In the case when $M$ is a sphere of radius 1

$$
I_{q}=g_{\text {phys }}^{-2 \alpha_{\tau} q} \exp \left(-\frac{8 \pi^{2} q}{g_{\text {phys }}^{2}}+\left(\frac{11}{6} \alpha_{\tau}-\frac{2}{3} \alpha_{T}\right) q \log v\right) \int_{R_{q}} \Phi(A) d(A) d v
$$

where

$$
\begin{aligned}
d(A) & =\left(\operatorname{det}^{\prime}\left(D_{A}-\mathscr{M}\right)^{2}\right)^{1 / 2}\left(\operatorname{det}^{\prime}\left(D_{0}-\mathscr{M}\right)^{2}\right)^{-1 / 2}, \\
d v & =\left(\frac{\operatorname{det}^{\prime} \Delta_{2}^{A}}{\operatorname{det}^{\prime} \Delta_{2}^{0}}\right)^{-1 / 2}\left(\frac{\operatorname{det}^{\prime} \Delta_{0}^{A}}{\operatorname{det}^{\prime} \Delta_{0}^{0}}\right)^{1 / 2}\left(\frac{\text { volume } H^{A}}{\text { volume } H^{0}}\right)^{-1} .
\end{aligned}
$$

The constant $K$ must be choosen from the requirement of coincidence of our regularization procedure with the usual one. However the value of this constant is inessential for us because we omit all constant factors in instanton contribution; therefore we can take $K=1$.

Let us consider the case when the fermion masses are small. Without loss of generality one can assume that the fermion fields $\psi(x)$ consists of the fermion fields $\psi_{1}(x), \ldots, \psi_{N}(x)$ transforming according irreducible representations $V_{1}, \ldots, V_{N}$ and the mass matrix $\mathscr{M}$ is diagonal, i.e. the fermion part of Lagrangian has the form

$$
\sum_{1 \leqq j \leqq N} \psi_{j} D_{j}^{A} \psi_{j}-\sum_{1 \leqq j \leqq N} m_{j} \bar{\psi}_{j} \psi_{j}
$$

where $D_{j}^{A}$ is the Dirac operator in the instanton field $A$ acting on the fermions transforming according the representation $V_{j}$. Let us denote by $\delta_{j}$ the number of zero modes of the operator $\mathrm{D}_{j}^{A}$. In the case of small fermion masses $m_{j}$ one can approximate $\operatorname{det}^{\prime}\left(D_{j}^{A}-m_{j}\right)$ by $\operatorname{det}^{\prime}\left(D_{j}^{A}\right) \cdot m_{j}^{\delta_{j}}$. Therefore

$$
\operatorname{det}^{\prime}\left(D_{A}-\mathscr{M}\right)=m_{1}^{\delta_{1}} \ldots m_{N}^{\delta_{N}} \operatorname{det}^{\prime} D_{A} .
$$

To check this assertion we divide $\log \operatorname{det}^{\prime}\left(D_{j}^{A}-m_{j}\right)$ on two parts : the contribution of eigenvalues which have nonzero limit for $m_{j} \rightarrow 0$ and the contribution of eigenvalues tending to zero for $m_{j} \rightarrow 0$. The first part give $\log \operatorname{det}^{\prime} D_{j}^{A}$ in the $\operatorname{limit}$ $m_{j} \rightarrow 0$. The eigenvalues of second kind can be calculated by means of perturbation theory; we see that there are $\delta_{j}$ eigenvalues of second kind approximately equal to $-m_{j}$ and therefore their contribution is equal to $\delta_{j} \log m_{j}$.

\section{Section 8. Conformal Properties of Instanton Contribution}

The Yang-Mills action is conformally invariant. However the determinants arising by calculation of instanton contribution do not admit conformal invariance, because the renormalization disturbes the conformal invariance of quantum theory. Nevertheless one can analyse the behaviour of instanton determinants by conformal transformation and obtain from this analysis information on the instanton contribution. 
Let us consider the variation of the expression

$F=\frac{1}{2} \log \operatorname{det}^{\prime} \Delta_{2}^{A}-\frac{1}{2} \log \operatorname{det}^{\prime} \Delta_{0}^{A}$

by conformal variation of riemannian metric

$$
g_{\alpha \beta}^{\prime}(x)=\exp (\sigma(x)) g_{\alpha \beta}(x) .
$$

(All fields are defined on compact manifold $M$.) The variation of scalar product $\left(\omega_{1}, \omega_{2}\right)$ by infinitesimal conformal transformation is equal to

$$
(2-k)\left(\hat{\sigma} \omega_{1}, \omega_{2}\right)
$$

if $\omega_{1}, \omega_{2}$ are $k$-forms.

The variation $\left(d_{A}^{*}\right)^{*}$ of operator $d_{A}^{*}=-* d_{A} *$ on the $k$-forms by this transformation is given by the formula

$$
\left(d_{A}^{*}\right)^{\cdot}=(k-3) \hat{\sigma} d_{A}^{*}+(2-k) d_{A}^{*} \hat{\sigma}
$$

[here $\hat{\sigma}$ is the operator of multiplication on the function $\sigma(x)$ ]. The operator $P=\frac{1}{2}(1-*)$ on 2 -forms is conformally invariant. Therefore the variations of the operators $\Delta_{2}^{A}=P d_{A} d_{A}^{*} P, \Delta_{0}^{A}=d_{A}^{*} d_{A}$ can be represented in the form

$$
\begin{aligned}
& \left(\Delta_{2}^{A}\right)^{\cdot}=-P d_{A} \hat{\sigma} d_{A}^{*} P, \\
& \left(\Delta_{0}^{A}\right)^{*}=-2 \hat{\sigma} d_{A}^{*} d_{A}+d_{A}^{*} \hat{\sigma} d_{A} .
\end{aligned}
$$

From (4), (5) we obtain that the variation of the expression

$$
V(t)=\frac{1}{2} \operatorname{Sp} \exp \left(-t \Delta_{2}^{A}\right)-\frac{1}{2} \operatorname{Sp} \exp \left(-t \Delta_{0}^{A}\right)
$$

by infinitesimal conformal transformation is equal to

$$
\begin{aligned}
\dot{V}(t)= & -\frac{t}{2} \operatorname{Sp}\left(\left(\Delta_{2}^{A}\right)^{\cdot} \exp \left(-t \Delta_{2}^{A}\right)\right)+\frac{t}{2} \operatorname{Sp}\left(\left(\Delta_{0}^{A}\right)^{\cdot} \exp \left(-t \Delta_{0}^{A}\right)\right) \\
= & \frac{t}{2} \operatorname{Sp}\left(\hat{\sigma} d_{A}^{*} P d_{A} \exp \left(-t \Delta_{1}^{A}\right)-t \operatorname{Sp}\left(\hat{\sigma} d_{A}^{*} d_{A} \exp \left(-t \Delta_{0}^{A}\right)\right)\right. \\
& +\frac{t}{2} \operatorname{Sp}\left(\hat{\sigma} d_{A} d_{A}^{*} \exp \left(-t \Delta_{1}^{A}\right)\right. \\
= & \frac{t}{2} \operatorname{Sp}\left(\hat{\sigma} \Delta_{1}^{A} \exp \left(-t \Delta_{1}^{A}\right)\right)-t \operatorname{Sp}\left(\hat{\sigma} \Delta_{0}^{A} \exp \left(-t \Delta_{0}^{A}\right)\right) \\
= & -\frac{1}{2} t \frac{d}{d t}\left(\operatorname{Sp}\left(\hat{\sigma} \exp \left(-t \Delta_{1}^{A}\right)\right)-2 \operatorname{Sp}\left(\hat{\sigma} \exp \left(-t \Delta_{0}^{A}\right)\right)\right)
\end{aligned}
$$

where $\Delta_{1}^{A}=d_{A} d_{A}^{*}+d_{A}^{*} P d_{A}$ as earlier. [We have used the relations

$$
\begin{aligned}
\Delta_{2}^{A} P d_{A} & =P d_{A} \Delta_{1}^{A}, \exp \left(-t \Delta_{2}^{A}\right) P d_{A}=P d_{A} \exp \left(-t \Delta_{1}^{A}\right), \\
\Delta_{0}^{A} d_{A}^{*} & =d_{A}^{*} \Delta_{1}^{A}, \exp \left(-t \Delta_{0}^{A}\right) d_{A}^{*}=d_{A}^{*} \exp \left(-t \Delta_{1}^{A}\right)
\end{aligned}
$$

by calculation of $V(t)$.] 
It follows from (7) that the variation $\dot{\Phi}_{k}$ of the expressions $\Phi_{k}=\frac{1}{2} \Phi_{k}\left(\Delta_{2}^{A}\right)$ $-\frac{1}{2} \Phi_{k}\left(\Delta_{0}^{A}\right)$, connected with the asymptotics of $V(t)$ for $t \rightarrow 0$, is given by

$$
\dot{\Phi}_{k}=\frac{1}{2} k\left(\Psi_{k}\left(\hat{\sigma} \mid \Delta_{1}^{A}\right)-2 \Psi_{k}\left(\hat{\sigma} \mid \Delta_{0}^{A}\right) .\right.
$$

Using (6.5) one can express $F$ through $V(t)$

$$
\begin{aligned}
F= & -\int_{0}^{\infty} t^{-1}\left(V(t)-\Phi_{2} t^{-2}-\Phi_{1} t^{-1}\right. \\
& \left.-\theta(1-t) \Phi_{0}-\theta(t-1)\left(\frac{1}{2} h_{2}^{A}-\frac{1}{2} h_{0}^{A}\right)\right) d t .
\end{aligned}
$$

Now we can obtain the following expression for variation of $F$ by infinitesimal conformal transformation of metric:

$$
\dot{F}=-\frac{1}{2}\left(\Psi_{0}\left(\hat{\sigma} \mid \Delta_{1}^{A}\right)-\operatorname{Sp} \hat{\sigma} \Pi\left(\Delta_{1}^{A}\right)\right)+\left(\Psi_{0}\left(\hat{\sigma} \mid \Delta_{0}^{A}\right)-\operatorname{Sp} \hat{\sigma} \Pi\left(\Delta_{0}^{A}\right)\right) .
$$

To derive (10) we represent $F$ in the form

$$
\begin{aligned}
\dot{F}= & -\int_{0}^{\infty} t^{-1}\left(\dot{V}(t)-\dot{\Phi}_{2} t^{-2}-\dot{\Phi}_{1} t^{-1}\right) d t \\
= & \frac{1}{2} \int_{0}^{\infty} \frac{d}{d t}\left(\operatorname{Sp} \hat{\sigma} \exp \left(-t \Delta_{1}^{A}\right)-\Psi_{2}\left(\hat{\sigma} \mid \Delta_{1}^{A}\right) t^{-2}-\Psi_{1}\left(\hat{\sigma} \mid \Delta_{0}^{A}\right) t^{-1}\right) d t \\
& -\int_{0}^{\infty} \frac{d}{d t}\left(\operatorname{Sp} \hat{\sigma} \exp \left(-t \Delta_{0}^{A}\right)-\Psi_{2}\left(\hat{\sigma} \mid \Delta_{0}^{A}\right) t^{-2}-\Psi_{1}\left(\hat{\sigma} \mid \Delta_{0}^{A}\right) t^{-1}\right) d t
\end{aligned}
$$

combining (7)-(9). The asymptotics of the integrands $t \rightarrow 0$ are given by Seeley formula and the asymptotics for $t \rightarrow \infty$ is governed by zero modes:

$$
\lim _{t \rightarrow \infty} \operatorname{Sp} \hat{\sigma} \exp \left(-t \Delta_{i}^{A}\right)=\operatorname{Sp} \hat{\sigma} \Pi\left(\Delta_{i}^{A}\right) .
$$

Using these asymptotics and (11) we get (10).

To find the variation of the volume of the isotropy subgroup $H^{A}$ by the conformal variation of the metric in $M$ we must study the variation of the measure in the Lie algebra of $H^{A}$. This Lie algebra can be realized as $\operatorname{Ker} \Delta_{0}^{A} \subset \Gamma_{0}$. If the new scalar product in euclidean space is expressed through the old one by the formula $\langle f, g\rangle=(B f, g)$ then the new measure can be obtained from the old one by multiplication on $(\operatorname{det} B)^{1 / 2}$. If $B=1+\varepsilon S, \varepsilon \rightarrow 0$, then $(\operatorname{det} B)^{1 / 2} \approx 1+\frac{1}{2} \varepsilon \operatorname{Sp} S$ $=1+\frac{1}{2} \varepsilon \sum\left(S f_{i}, f_{i}\right)$, where $f_{i}$ run over the orthonormal basis. Using these well known assertions and (3) we can calculate the variation of the measure in $\operatorname{Ker} \Delta_{0}^{A}$ by infinitesimal conformal transformation [the operator $2 \hat{\sigma}$ plays the role of operator $\varepsilon S$ and $\left.\frac{1}{2} \varepsilon \sum\left(S f_{i}, f_{i}\right)=\frac{1}{2} \operatorname{Sp} 2 \hat{\sigma} \Pi\left(\Delta_{0}^{A}\right)\right]$. Therefore the variation of (volume $\left.H^{A}\right)^{-1}$ is equal to

$$
\operatorname{Sp} \hat{\sigma} \Pi\left(\Delta_{0}^{A}\right)\left(\text { volume } H^{A}\right)^{-1} \text {. }
$$

Let us consider now the manifold $R$ obtained from the manifold of regular instantons $N$ by means of identification of gauge equivalent instantons and the measure $d \mu_{0}$ induced by the natural riemannian metric in $R$. The variation of the measure $d \mu_{0}$ by infinitesimal conformal variation of the metric in $M$ is equal to

$$
\frac{1}{2} \operatorname{Sp}\left(\hat{\sigma} \Pi\left(\Delta_{1}^{A}\right)\right) d \mu_{0} .
$$


To prove this assertion we note that the tangential space to the manifold $N$ can be identified with the space of solutions of linearized duality equation; in other words with the space $\operatorname{Ker} P d_{A}$ where $P d_{A}$ acts from $\Gamma_{1}$ into $\Gamma_{2}$. The tangential space to the manifold $R$ can be identified with the coset space $\operatorname{Ker} P d_{A} / \operatorname{Im} d_{A}$. If $a \in \operatorname{Ker} P d_{A}, b \in \operatorname{Ker} P d_{A}$ then the scalar product of corresponding elements $\tilde{a}, \tilde{b}$ of $\operatorname{Ker} P d_{A} / \operatorname{Im} d_{A}$ is equal to $\left(\Pi\left(\Delta_{1}^{A}\right) a, \Pi\left(\Delta_{1}^{A}\right) b\right)$. In other words the space $\operatorname{Ker} P d_{A} / \operatorname{Im} d_{A}$ is isometric to the space $\operatorname{Ker} \Delta_{1}^{A}$ [this isometry follows from the Hodge decomposition theorem applied to the elliptic complex (4.13)]. The conformal transformation of riemannian metric in $M$ does not change the manifold $R$ but this transformation changes the riemannian metric in $R$. If the element $\tilde{a}$ of tangential space $T(R)$ corresponds to the element $a \in \operatorname{Ker} \Delta_{1}^{A}$ then the new length of $\tilde{a}$ is equal to $\left(\exp (\hat{\sigma}) \Pi\left(\Delta_{1}^{\prime A}\right) a, \Pi\left(\Delta_{1}^{\prime A}\right) a\right)^{1 / 2}$ where $\Delta_{1}^{\prime A}$ is the operator $\Delta_{1}^{A}$ in the new metric. Hence the variation of the length of $\tilde{a}$ by the infinitesimal conformal transformation is given by the formula $\frac{1}{2}(\hat{\sigma} a, a)$. We see that the new measure in $T(R)$ can be obtained from the old one by means of multiplication on

$$
1+\frac{1}{2} \sum\left(\hat{\sigma} a_{i}, a_{i}\right)=1+\frac{1}{2} \operatorname{Sp} \hat{\sigma} \Pi\left(\Delta_{1}^{A}\right)
$$

where $a_{i}$ denotes the orthonormal basis in $\Pi\left(\Delta_{1}^{A}\right)$. This proves the formula (13).

Let us consider now the measure $d \mu=\gamma(A) d \mu_{0}$ on the manifold $N$ where

$$
\gamma(A)=\left(\operatorname{det}^{\prime} \Delta_{2}^{A}\right)^{-1 / 2}\left(\operatorname{det}^{\prime} \Delta_{0}^{A}\right)^{1 / 2}\left(\text { volume } H^{A}\right)^{-1}
$$

Combining the formulae (10)-(13), we get the following assertions.

Theorem 4. The variation of the measure $d \mu$ by the infinitesimal conformal transformation of the riemannian metric in $M$ is equal to

$$
\left(\frac{1}{2} \Psi_{0}\left(\hat{\sigma} \mid \Delta_{1}^{A}\right)-\Psi_{0}\left(\hat{\sigma} \mid \Delta_{0}^{A}\right)\right) d \mu .
$$

The variation of $\gamma(0)$ is equal to

$$
\frac{1}{2} \Psi_{0}\left(\hat{\sigma} \mid \Delta_{1}^{0}\right)-\Psi_{0}\left(\hat{\sigma} \mid \Delta_{0}^{0}\right)
$$

The conformal variation of the measure

$$
d v=\frac{\gamma(A)}{\gamma(0)} d \mu_{0}
$$

entering in the expression for instanton contribution can be obtained from Theorem 4.

Theorem 4'. If the riemannian metric in $M$ is replaced by the conformally equivalent metric $g_{\alpha \beta}^{\prime}(X)=\exp (\sigma(x)) g_{\alpha \beta}(x)$ then the measure $d v^{\prime}$ on the manifold $R$ corresponding to the metric $g_{\alpha \beta}^{\prime}$ is given by the formula

$$
d v^{\prime}=\exp \left(\frac{11}{192} \alpha_{\tau} \int \sigma(x)\left\langle F_{\mu v}, F^{\mu v}\right\rangle d V\right) d v,
$$

where $\alpha_{\tau}$ denotes Dynkin index of adjoint representation.

To deduce Theorem $4^{\prime}$ from Theorem 4 we note that the variation of $d v$ by infinitesimal conformal transformation is given by

$$
\begin{aligned}
& \left(\frac{1}{2} \Psi_{0}\left(\hat{\sigma} \mid \Delta_{1}^{A}\right)-\Psi_{0}\left(\hat{\sigma} \mid \Delta_{0}^{A}\right)-\frac{1}{2} \Psi_{0}\left(\hat{\sigma} \mid \Delta_{1}^{0}\right)+\Psi_{0}\left(\hat{\sigma} \mid \Delta_{0}^{0}\right)\right) d v \\
& \quad=\left(\frac{11}{192} \alpha_{\tau} \int \sigma(x)\left\langle F_{\mu \nu}, F^{\mu \nu}\right\rangle d V\right) d v
\end{aligned}
$$


as follows from (14), (15) and from calculations by means of Seeley formula. Using the conformal invariance of (17) we obtain from (17) the expression (16) for variation of $d v$ by finite conformal transformation.

Let us consider now conformal properties of the determinant of Dirac operator $D_{A}$. One can prove that

$$
\exp \left(\frac{5}{4} \hat{\sigma}\right) D_{A}^{\prime}=D_{A} \exp \left(\frac{3}{4} \hat{\sigma}\right)
$$

where $D_{A}^{\prime}$ is the Dirac operator corresponding to the metric $g_{\alpha \beta}^{\prime}(x)=\exp (\sigma(x)) g_{\alpha \beta}(x)$. [The usual proof of conformal invariance of the Dirac equation $D_{A} \psi=0$ is based on (18); see [39] for example.] It follows from (18) that the variation $\dot{D}_{A}$ of the Dirac operator by the infinitesimal conformal transformation of the metric is given by the formula

$$
\dot{D}_{A}=-\frac{5}{4} \hat{\sigma} D_{A}+\frac{3}{4} D_{A} \hat{\sigma} \text {. }
$$

The conformal variation of $W(t)=\operatorname{Sp} \exp \left(-D_{A}^{2} t\right)$ is equal to

$$
\begin{aligned}
\dot{W}(t) & =-t \operatorname{Sp}\left(\left(\dot{D}_{A} D_{A}+D_{A} \dot{D}_{A}\right) \exp \left(-t D_{A}^{2}\right)\right) \\
& =-t \frac{d}{d t} \operatorname{Sp}\left(\hat{\sigma} \exp \left(-D_{A}^{2} t\right)\right) .
\end{aligned}
$$

Now we can study the variation $\dot{G}$ of

$$
\begin{aligned}
G= & \log \operatorname{det}^{\prime} D_{A}=\frac{1}{2} \log \operatorname{det}^{\prime} D_{A}^{2} \\
= & -\frac{1}{2} \int_{0}^{\infty} t^{-1}\left(W(t)-\Phi_{2}\left(D_{A}^{2}\right) t^{-2}-\Phi_{1}\left(D_{A}^{2}\right) t^{-1}-\Phi_{0}\left(D_{A}^{2}\right) \theta(1-t)\right. \\
& \left.-\operatorname{Sp} \Pi\left(D_{A}^{2}\right) \theta(t-1)\right) d t .
\end{aligned}
$$

We obtain that

$$
\begin{aligned}
\dot{G} & =-\frac{1}{2} \int_{0}^{\infty} t^{-1}\left(\dot{W}(t)-\dot{\Phi}_{2}\left(D_{A}^{2}\right) t^{-2}-\dot{\Phi}_{1}\left(D_{A}^{2}\right) t^{-1}\right) d t \\
& =\frac{1}{2} \int_{0}^{\infty} \frac{d}{d t}\left(\operatorname{Sp}\left(\hat{\sigma} \exp \left(-D_{A}^{2} t\right)\right)-\Psi_{2}\left(\hat{\sigma} \mid D_{A}^{2}\right) t^{-2}-\Psi_{1}\left(\hat{\sigma} \mid D_{A}^{2}\right) t^{-1}\right) d t
\end{aligned}
$$

and therefore

$$
\dot{G}=-\frac{1}{2} \Psi_{0}\left(\hat{\sigma} \mid D_{A}^{2}\right)+\frac{1}{2} \operatorname{Sp} \hat{\sigma} \Pi\left(D_{A}^{2}\right) .
$$

The expression

$$
\begin{aligned}
f(A) & =\operatorname{det}^{\prime} D_{A}\left(\operatorname{det}^{\prime} D_{0}\right)^{-1} \\
& =\exp \left(\frac{1}{2} \log \operatorname{det}^{\prime} D_{A}^{2}-\frac{1}{2} \log \operatorname{det}^{\prime} D_{0}^{2}\right)
\end{aligned}
$$

enters in the instanton contribution in the euclidean Green functions.

Theorem 5. The variation of $f(A)$ by infinitesimal conformal variation of the metric is equal to

$$
\dot{f}(A)=\left(-\frac{1}{48 \pi^{2}} \alpha_{T} \int \sigma(x)\left\langle F_{\mu \nu}, F^{\mu \nu}\right\rangle d V+\frac{1}{2} \operatorname{Sp} \hat{\sigma} \Pi\left(D_{A}^{2}\right)\right) f(A)
$$

(the fermions transform according the representation $T, \alpha_{T}$ denotes Dynkin index). 
To prove this assertion one must use (21) and calculate RHS of (21) by means of Seeley formula.

In the proofs of Theorems 4 and 5 some ideas of [25] are used. The physical origin of theorems proved in present section is clear: the local formulae for conformal variations can be obtained because the breaking of conformal invariance is a renormalization effect. We have shown in $[26,27]$ that the theorems of $[25,40]$ have similar origin. One can prove general assertions including the results of this section and the results of $[25,40]$ (see $[26,27]$ ).

Let us consider now the instanton contribution in the case $M=S^{4}$. The 15 -parameter group $K$ of conformal transformation acts on $S^{4}$. It follows from conformal invariance of duality equation that the group $K$ acts on the manifold $R$. The variation of instanton determinants by the conformal transformations of the sphere can be studied by means of theorems proved in present section. If $x^{\prime}=F(x)$ is a conformal transformation of the sphere then

$$
d s^{\prime 2}=\exp (\sigma(x)) d s^{2}
$$

and the variation of $d v$ and $f(A)$ is given by the formulae (16), (22) where $\sigma(x)$ is determined by (23). The density of the measure $d v$ and the function $f$ depend on $m$ parameters where $m=\operatorname{dim} R=h_{1}^{A}$. The conformal properties mentioned above permit to express these functions on the orbit of the group $K$ in $R$ through the values in one point of the orbit. Therefore these functions can be expressed through an arbitrary function depending on $m-d$ variables, where $d$ denotes the dimension of the orbit of the group $K$ in $R$. In other words each of these functions can be represented as a product of knwon function and conformally invariant function on $R$. For example in the case $G=\mathrm{SU}(2)$ the manifold $R$ is 13-dimensional and the orbits of the group $K$ in $R$ are 12-dimensional, therefore the instanton contribution can be expressed through an arbitrary function depending on one variable. In the case $G=\mathrm{SU}(2), q=1$ we have $m=d=5$ and only a constant factor cannot be determined by means of conformal properties.

\section{Section 9. Two-Instanton Contribution}

In this section we explain briefly how the results of the preceding section can be used to get information on the two-instanton contribution in the euclidean Green functions in the case $G=\mathrm{SU}(2), q=2$. The results of present section are obtained in collaboration with Frolov [42].

We are interested in the instantons in euclidean space. However it is convenient to represent the euclidean space as a sphere of infinite radius. In other words to find the instanton contribution in euclidean space we can study the contribution of spherical instantons having small size with respect to the radius of the sphere. In our case every instanton is gauge equivalent to the t'Hooft instanton:

$$
\begin{aligned}
A_{\mu}^{a} & =\eta_{\mu \nu}^{a} \partial_{v} \log \varrho, \\
\varrho & =\frac{\lambda_{1}^{2}}{\left(x-y_{1}\right)^{2}}+\frac{\lambda_{2}^{2}}{\left(x-y_{2}\right)^{2}}+\frac{\lambda_{3}^{2}}{\left(x-y_{3}\right)^{2}},
\end{aligned}
$$


where $\lambda_{1}, \lambda_{2}, \lambda_{3}$ are positive numbers, $y_{1}, y_{2}, y_{3}$ are four-dimensional vectors (see $[7,43])$. The t'Hooft solution does not change if $\lambda_{i}$ is replaced by $k \lambda_{i}$ and therefore depends on 14 parameters. One of parameters is connected with the gauge freedom [7]. The conformal group $K$ acts on the manifold of parameters $y_{1}, y_{2}, y_{3}, \lambda_{1}, \lambda_{2}, \lambda_{3}$. There exist two independent conformally invariant combinations of instanton parameters

$$
u_{1}=c_{1} c_{3}^{-1}, \quad u_{2}=c_{2} c_{3}^{-1}
$$

where

$$
c_{1}=\lambda_{1}^{2}\left(y_{2}-y_{3}\right)^{2}, \quad c_{2}=\lambda_{2}^{2}\left(y_{1}-y_{3}\right)^{2}, \quad c_{3}=\lambda_{3}^{2}\left(y_{1}-y_{2}\right)^{2} .
$$

The expression

$$
u=u_{1} u_{2}\left(1+u_{1}+u_{2}\right)^{-3}
$$

is also gauge invariant. One can exclude the gauge freedom imposing the conditions $u_{2}=1,0<u_{1}<1$.

To study the instanton contribution in euclidean space we must calculate the function $f(A)$ and the measure $d v$ only for instantons satisfying $\lambda_{i} \ll R,\left|y_{i}\right| \ll R$, where $R$ denotes the radius of the sphere.

We restrict ourselves by the case when the gauge fields interact with $N$ fermion isodoublets having small masses. Using the results of Sect. 8 and the zero fermion modes found in [14] we obtain the following expression for two-instanton contribution:

$$
\begin{aligned}
& g_{\text {phys }}^{-16} \int \Phi(\lambda, y) \\
& \cdot \exp \left(-\frac{16 \pi^{2}}{g_{\text {phys }}^{2}}+\left(\frac{22}{3}-\frac{2}{3} N\right) \log v^{2} p^{2}\right) \prod_{l=1}^{N}\left(m_{l} p\right)^{2} d \mu_{\text {inv }},
\end{aligned}
$$

where

$$
p^{2}=\left(c_{1} c_{2} c_{3}\right)^{1 / 2}\left(c_{1}+c_{2}+c_{3}\right)^{-1 / 2}\left(\lambda_{1}^{2}+\lambda_{2}^{2}+\lambda_{3}^{2}\right)^{-1}
$$

$d \mu_{\mathrm{inv}}$ is conformally invariant measure:

$$
d \mu_{\mathrm{inv}}=F \cdot\left(\prod_{k \neq j}\left(y_{k}-y_{j}\right)^{2}\right)^{-1} \prod_{i} \lambda_{i}^{-2} d \lambda_{i}^{2} d y_{i} .
$$

$F$ is a conformally invariant function. [This function can be taken in the form $\varphi\left(u_{1}\right) \delta\left(u_{2}-1\right) \delta\left(\lambda_{1}^{2}+\lambda_{2}^{2}+\lambda_{3}^{2}-1\right)$ where $\varphi$ is an arbitrary function and the factor $\delta\left(u_{2}-1\right)$ corresponds to the gauge condition $u_{2}=1$. The integral (1) reduces in this case to the 13-dimensional integral.] It is easy to check that $p^{2}$ is gauge invariant. In the case when the instanton can be considered as superposition of 
two distant instantons having topological number $1 p^{2}$ can be interpreted as product of sizes of these two instantons. (One can verify that for such instantons $u \approx 0$.)

The methods of Sects. 8 and 9 permit to calculate completely the instanton contribution in two-dimensional non-linear $\sigma$-model [44].

\section{Appendix I}

The asymptotics for $t \rightarrow 0$ of $\operatorname{Sp} Q \exp (-K t)$, where $Q$ is the operator of multiplication on the matrix function $Q(x)=\left(Q_{j}^{i}(x)\right)$, were calculated by V. Romanov for all operators used in present paper [i.e. in the cases $K=\Delta_{0}^{A}, \Delta_{1}^{A}, \Delta_{2}^{A},\left(D_{A}-\mathscr{M}\right)^{2}$ ] and for other operators playing the role in quantum field theory [45]. In the cases under consideration

$$
\operatorname{Sp} Q \exp (-K t) \approx \Psi_{2}(Q \mid K) t^{-2}+\Psi_{1}(Q \mid K) t^{-1}+\Psi_{0}(Q \mid K) .
$$

It is convenient to represent $\Psi_{k}(Q \mid K)$ in the form

$$
\Psi_{k}(Q \mid K)=\int Q_{j}^{i}(x) \Psi_{k}(x \mid K)_{i}^{j} d V,
$$

where $\psi_{k}(x \mid K)=\left(\psi_{k}(x \mid K)_{i}^{j}\right)$ is a matrix function, $d V$ denotes the riemannian volume element. [The operator $K$ acts in the space of sections $\Gamma(\xi)$ of vector bundle $\xi(E, M, F, p)$. The matrix functions $Q(x)$ and $\psi_{k}(x \mid K)$ depend on the choice of local coordinates in the riemannian manifold $M$ and of the local trivialization of $\xi$, but the integrand in (1) does not depend on this choice.]

For example one can check that in the case $K=D_{A}^{2}$

$$
\begin{aligned}
\psi_{2}\left(x \mid D_{A}^{2}\right)= & 1 \\
\psi_{1}\left(x \mid D_{A}^{2}\right)= & -\frac{1}{12} R-\frac{1}{2}\left(\gamma^{a} \gamma^{b}-\gamma^{b} \gamma^{a}\right) t\left(F_{a b}\right), \\
\psi_{0}\left(x \mid D_{A}^{2}\right)= & \frac{1}{288} R^{2}-\frac{1}{180} R_{a b} R_{a b}-\frac{7}{1440} R_{a b c d} R_{a b c d} \\
& -\frac{1}{120} \square R+\frac{1}{192} \varepsilon_{a b c d} R_{a b i k} R_{c d i k} \gamma^{5} \\
& +\frac{1}{2}\left(\gamma^{a} \gamma^{b}-\gamma^{b} \gamma^{a}\right)\left[\frac{1}{24} R_{a b c d} t\left(F_{c d}\right)-\frac{1}{24} R t\left(F_{a b}\right)+\frac{1}{18} R_{c a} t\left(F_{c b}\right)\right] \\
& -\frac{1}{12} t\left(F_{a b}\right) t\left(F_{a c}\right) \gamma^{b} \gamma^{c}+\frac{1}{8} t\left(F_{a b}\right) t\left(F_{c d}\right) \gamma^{a} \gamma^{b} \gamma^{c} \gamma^{d} .
\end{aligned}
$$

We assume that the fermions transform according the representation $T$ of the gauge group $G$; the corresponding representation of the Lie algebra is denoted by $t$. We use the Vierbein formalism; for instance, $F_{a b}=F^{a b}=F_{\mu \nu} l_{a}^{\mu} l_{b}^{\nu}$, where $l_{a}^{\mu}(x)$ denotes the Vierbein (orthonormal frame) at the point $x$. The expression $t\left(F_{a b}\right)$ is considered as a matrix with respect to the isotopic indices.

The coefficients $\Psi_{k}(Q \mid K)$ can be used for calculation of one-loop renormalization and anomalies. Conversely, partial information on these coefficients can be extracted from well known results concerning anomalies; this information is sufficient to derive the assertions of present paper. 
The index of the Dirac operator considered only on the right spinors can be represented as $\Psi_{0}\left(\gamma^{5} \mid D_{A}^{2}\right)$ [this follows from the formula (3.3) proved in Sect. 3]. Hence this index can be obtained from (2). The index of the operator $T$ defined in Sect. 4 is equal to $\Phi_{0}\left(\Delta_{2}^{A}\right)+\Phi_{0}\left(\Delta_{0}^{A}\right)-\Phi_{0}\left(\Delta_{1}^{A}\right)$ and therefore can be calculated in a similar way. However there exists a very simple method of calculation of index in the cases under consideration. Namely, the symbols of our operators are generated by universal construction (see [32] for example) and therefore its indices can be expressed through the characteristic classes. It is too lengthy to describe the expression for index in general case and therefore we restrict ourselves by the special case which is sufficient for the aims of the present paper. Let us consider the elliptic operator $K$ transforming the field $\varphi_{1}$ into the field $\varphi_{2}$. We suppose that the fields $\varphi_{1}$ and $\varphi_{2}$ are defined on four-dimensional oriented riemannian manifold $M$ and have isotopic and spatial indices. The isotopic indices transform according the $N$-dimensional representation $V$ of simple non-abelian compact Lie group $G$, the spatial indices of $\varphi_{i}$ transform according the $L$-dimensional representation $W_{i}$ of $\mathrm{SO}(4)$. In other words the field $\varphi_{i}$ can be considered as a section of the vector bundle $\xi_{V \otimes W_{i}}$ associated with principal fibration $\xi(E, M, G \times \mathrm{SO}(4), p)$, which is defined as product of principal fibration $\eta\left(E_{\eta}, M, G, p_{\eta}\right)$ and tangential fibration $\zeta\left(E_{\zeta}, M, \mathrm{SO}(4), p_{\zeta}\right)$. [The tangential fibration of oriented riemannian manifold can be considered as principal fibration with the group $\mathrm{SO}(4)$. The representation $V \otimes$ $W_{i}$ of $G \times \mathrm{SO}(4)$ is defined as tensor product of $V$ and $\left.W_{i}\right]$ We assume that the symbol of the operator $K$ is $G \times \mathrm{SO}(4)$-invariant in the natural sense. Then one can express the index of operator $K$ through the weights of representations $V, W_{1}, W_{2}$ and topological numbers of fibrations $\eta$. $气$. The group $\mathrm{SO}(4)$ is locally isomorphic to the product of two simple groups SU(2). Therefore the tangential fibration can be characterized by two topological numbers; these numbers can be expressed through the Euler characteristic

$$
\chi(M)=\frac{1}{128 \pi^{2}} \int \varepsilon^{\mu \nu \alpha \beta} \varepsilon^{\lambda Q \gamma \delta} R_{\mu \nu \lambda \varrho} R_{\alpha \beta \gamma \delta} d V
$$

and the signature of the manifold $M$

$$
\tau(M)=\frac{1}{96 \pi^{2}} \int \varepsilon^{\mu \nu \alpha \beta} R_{\mu \vee \lambda \varrho} R_{\alpha \beta}^{\lambda \varrho} d V .
$$

Namely, the index of the operator $K$ can be represented in the form:

$$
\text { index } K=a \alpha_{V} q+N b \chi(M)-N\left(3 c-\frac{a}{4}\right) \tau(M) .
$$

Here $q$ denotes the topological number of the fibration $\eta, \alpha_{V}$ denotes the Dynkin index of the representation $V$.

The numbers $a, b$, and $c$ are defined by the formulae

$$
\begin{aligned}
& \sum v_{i}^{2}-\sum v_{i}^{\prime 2}=2 a \sigma_{1} \sigma_{2}, \\
& \sum v_{i}^{4}-\sum v_{i}^{\prime 4}=24 \sigma_{1} \sigma_{2}\left(b \sigma_{1} \sigma_{2}+c\left(\sigma_{1}^{2}+\sigma_{2}^{2}\right)\right),
\end{aligned}
$$


where $v_{i}, v_{i}^{\prime}$ denote the weights of representations $W_{1}, W_{2}$ respectively, the weights of vector representation of $\mathrm{SO}(4)$ are denoted by $\pm \sigma_{1}, \pm \sigma_{2}$. (The weights of representation are considered as linear functions on the Cartan subalgebra.) For example in the case when the operator $K$ coincide with the operator $T$ of Sect. 4 the representation $W_{1}$ is the vector representation and has the weights $\pm \sigma_{1}, \pm \sigma_{2}$ and the non-zero weights of the representation $W_{2}$ are equal to $\pm\left(\sigma_{1}-\sigma_{2}\right)$. Therefore in the case under consideration

$$
\begin{aligned}
& a=2, \quad b=-\frac{1}{2}, \quad c=\frac{1}{3} \\
& \text { index } T=2 \alpha_{\tau} q-\frac{1}{2} \operatorname{dim} G(\chi(M)+\tau(M)) .
\end{aligned}
$$

The index of operator $T$ was calculated in [8] by means of this method. We have mentioned in [8] also that if it is known that the index of $T$ has the form $\lambda q+\varrho \chi+\omega \tau$ then the coefficients $\lambda, \varrho$ can be expressed through the values of index $T$ for $M=S^{4}, q=0$ and $q=1$. This approach was used in [15].

If $K$ is the Dirac operator considered only on the right spinors then $a=1, b=0$, $c=\frac{1}{24}$. We obtain Theorem 2 of Sect. 5 .

\section{Appendix II}

\section{Integration of Invariant Functions}

Let $T$ be a homomorphism of compact Lie group $G$ in the isometry group of riemannian manifold $M$. We consider the integral

$$
\int_{M} f(x) d \mu,
$$

where $f(x)$ is a $G$-invariant function on $M$ [i.e. $f(T(g) x)=f(x)]$ and $d \mu$ is the measure on $M$ induced by riemannian metric. The isotropy subgroup at the point $x \in M$ [i.e. the set of elements $G$ satisfying $T(h) x=x$ ] will be denoted by $H(x)$. The orbit of the point $x \in M$ [i.e. the set of points $T(g) x$ ] where $g \in G$ will be denoted by $N(x)$. It is well known [41] that one can find such $G$-invariant open subset $M_{1} \subset M$ that all isotropy subgroups at the points $x \in M_{1}$ are conjugate and the measure of $M-M_{1}$ is equal to zero. By consideration of (1) one can replace $M$ by $M_{1}$. We assume therefore without loss of generality that isotropy subgroups at the points $x \in M$ are conjugate. The space of orbits will be denoted by $V$; the distance between the orbits $N(x)$ and $N(y)$ can be defined as the distance between the nearest points of these orbits. In the case under consideration $V$ is a riemannian manifold and the identification map of $M$ onto $V$ is a locally trivial fibration (see [41] for instance). The measure on $V$ determined by the riemannian metric will be denoted by $d v$. The riemannian metric in $M$ induces the riemannian metric in $N(x)$; the volume of $N(x)$ with respect to this metric will be denoted by $n(x)$. The integral of $G$-invariant function can be transformed into integral over the space of orbits; namely

$$
\int_{M} f(x) d \mu=\int_{V} f(x) n(x) d v
$$


[the functions $f(x)$ and $n(x)$ are $G$-invariant and therefore can be considered as functions on $V]$.

Let us denote by $\mathscr{G}$ the Lie algebra of the group $G$. We fix a scalar product in $\mathscr{G}$; this product induces a left invariant riemannian metric in $G$ and we normalize the scalar product by condition that volume of $G$ is equal to 1 . The homomorphism $T$ generates a homomorphism of $\mathscr{G}$ into the algebra of vector fields in $M$; therefore for every $x \in M$ a linear map $\tau_{x}$ of $\mathscr{G}$ into tangential space at point $x \in M$ is determined. We denote by $D(x)$ the determinant of $\tau_{x}^{*} \tau_{x}$ (the operator $\tau_{x}^{*} \tau_{x}$ can be degenerate; in this case we define the determinant as product of non-zero eigenvalues). The map $\tau_{x}$ vanishes at the Lie algebra $\mathscr{H}(x)$ of the group $H(x)$ and determines therefore a linear map $\tilde{\tau}_{x}$ of $\mathscr{G} / \mathscr{H}(x)$ into tangential space at the point $x \in M$; the map $\tilde{\tau}_{x}$ is non-degenerate. It is easy to see that $D(x)$ is equal to the determinant of $\tilde{\tau}_{x}^{*} \tilde{\tau}_{x}$.

One can check that

$$
n(x)=(D(x))^{1 / 2}(\text { volume } H(x))^{-1}
$$

where volume of the isotropy subgroup $H(x)$ is calculated with respect to the riemannian metric in $G$ defined above. To prove (3) we consider the coset space $G / H(x)$ provided by the riemannian metric induced by the riemannian metric in $G$. The group $G$ acts transitively on the manifold $G / H(x)$. The manifold $N(x)$ is homeomorphic to $G / H(x)$; there exists a homeomorphism $q_{x}$ of $G / H(x)$ onto $N(x)$ commuting with the action of the group $G$ on these manifolds. Let $\omega_{1}$ be the exterior form defined by the volume element in $G / H(x)$; analogous form in $N(x)$ will be denoted by $\omega_{2}$. The homeomorphism $q_{x}$ transforms the form $\omega_{2}$ into the form $q_{x}^{*} \omega_{2}$ on $G / H(x)$. The forms $\omega_{1}, \omega_{2}$ and $q_{x}^{*} \omega_{2}$ are $G$-invariant. The $G$-invariant $m$-form on the homogeneous $m$-dimensional manifold is determined up to constant factor therefore $q_{x}^{*} \omega_{2}=K(x) \omega_{1}$. To find the constant $K$ we can consider the differential $\left(q_{x}\right)_{*}$ of the map $q_{x}$ at the point of $G / H(x)$ determined by the coset $H(x) \subset G$. The tangential space at this point of $G / H(x)$ can be identified with the space $\mathscr{G} / \mathscr{H}(x)$ where $\mathscr{H}(x)$ denotes as earlier the Lie algebra of $H(x)$ and the differential $\left(q_{x}\right)_{*}$ coincides with map $\tilde{\tau}_{x}$. From this fact we get

$$
K(x)=\left(\operatorname{det} \tilde{\tau}_{x}^{*} \tilde{\tau}_{x}\right)^{1 / 2}=D(x)^{1 / 2}
$$

Using the identities

$$
\begin{aligned}
& \text { volume } G / H(x)=\int_{G / H(x)} \omega_{1} \\
& \text { volume } N(x)=\int_{N(x)} \omega_{2}=\int_{G / H(x)} q_{x}^{*} \omega_{2}=K \int_{G / H(x)} \omega_{1}
\end{aligned}
$$

we obtain

$$
n(x)=\text { volume } N(x)=D(x)^{1 / 2}(\text { volume } G / H(x))
$$


To calculate the volume of $G / H(x)$ we can apply (2) to the case $f(x)=1, M=G$ [the space $G / H(x)$ can be considered as the space of orbits of the group $H(x)$ acting in $G$ on the right; all orbits have the same volume]. We obtain from (2) that

$$
\text { volume } G / H(x)=(\text { volume } H(x))^{-1} \text {. }
$$

Let us consider the most important case when the scalar product fixed in $\mathscr{G}$ is invariant. Then the corresponding riemannian metric in $G$ is both left invariant and right invariant. The volume of the isotropy subgroup $H(x)$ does not depend on $x$ in this case because all isotropy subgroups are conjugate to the fixed subgroup $H$. The determinant $D(x)$ is $G$-invariant in this case and can be considered as a function on the coset space $V$. We see that

$$
\int_{M} f(x) d \mu=(\text { volume } H)^{-1} \int f(x) D(x)^{1 / 2} d v .
$$

At last we study the asymptotics of the integral

$$
\int f(x) \exp \left(-\frac{1}{\alpha} g(x)\right) d^{n} x
$$

for $\alpha \rightarrow 0$. We suppose that $f(x)$ and $g(x)$ are smooth functions on $n$-dimensional euclidean space, $f(x)$ is finite, $g(x) \geqq \gamma$. The set of points where $g(x)=\gamma$ will be denoted by $M$; we assume that $M$ is non-degenerate $r$-dimensional critical manifold i.e. the matrix $\partial^{2} g / \partial x^{i} \partial x^{j}$ [the Hessian of the function $g(x)$ ] has $n-r$ nonzero eigenvalues. The product of these eigenvalues will be denoted by $\Delta(x)$.

One can check that the asymptotics of the integral (7) in the limit $\alpha \rightarrow 0$ has the form

$$
(\pi \alpha)^{(n-r) / 2} \exp \left(-\frac{1}{\alpha} \gamma\right) \int_{M} f(x) \Delta(x)^{-1 / 2} d \mu,
$$

where $d \mu$ is the measure in $M$ corresponding to riemannian metric induced by standard metric in euclidean space.

One can consider (8) as approximation to (7) given by method of steepest descent.

Let us suppose now that the functions $f(x)$ and $g(x)$ are $G$-invariant [here $G$ denotes a subgroup of $O(n)]$. Then the integrand in (8) is $G$-invariant and the integral (8) can be transformed into integral over the space $V$ of the orbits of the group $G$ in $M$. Using (6) we obtain the following assertion.

Lemma. The asymptotics of the integral (7) in the limit $\alpha \rightarrow 0$ has the form

$$
(\pi \alpha)^{(n-r) / 2} \exp \left(-\frac{1}{\alpha} \gamma\right) \int_{V} f(x) d v
$$

where $V$ is the space of orbits of the group $G$ in $M$,

$$
d v=\Delta(x)^{-1 / 2} D(x)^{1 / 2}(\text { volume } G)(\text { volume } H(x))^{-1} d v_{0},
$$


$d v_{0}$ denotes the measure in $V$ corresponding to the natural riemannian metric, $H(x)$ is the isotropy subgroup at point $x \in M, T(x)$ is the operator in the Lie algebra $\mathscr{G}$ of the group $G$ defined by formula

$$
\langle T(x) h, h\rangle=\langle h x, h x\rangle
$$

and $D(x)$ denotes the product of non-zero eigenvalues of $T(x)$ [in RHS of (10) the symbol $\langle$,$\rangle denotes the usual scalar product in euclidean space and in LHS of (10)$ this symbol denotes the invariant scalar product in $\mathscr{G}$; the volumes of $G$ and of $H(x)$ must be calculated with respect to invariant riemannian metric in $G$ corresponding to this scalar product in $G]$.

Acknowledgement. I am indebted to E. Bogomolny, I. Frolov, A. Polyakov, V. Romanov, M. Shubin, Yu. Tyupkin and S. Vishik for valuable discussions.

\section{References}

1. Belavin, A., Polyakov, A., Schwarz, A., Tyupkin, Yu.: Phys. Lett. B 59, 85-87 (1975)

2. 't Hooft, G. : Phys. Rev. D 14, 3432-3450 (1976)

3. Polyakov, A. : Nucl. Phys. B 120, 429-458 (1977)

4. Belavin, A., Polyakov, A. : Nucl. Phys. B 123, 429-444 (1977)

5. Witten, E.: Phys. Rev. Lett. 38, 121-124 (1977)

6. Burlakov, D., Dutyshev, V.: ZhETF 73, 377--381 (1977)

7. Jackiw, R., Nohl, C., Rebbi, C.: Phys. Rev. D 15, 1642-1646 (1977)

8. Schwarz, A.: Phys. Lett. B 167, 172-174 (1977)

9. Schwarz, A.: In: Proc. of Symp. in Alushta (USSR), April 1976, JINR preprint D2-9788, p. 224-242

10. Jackiv, R., Rebbi, G.: Phys. Lett. B 67, 189-192 (1977)

11. Atiyah, M., Hithin, N., Singer, I.: Proc. Nat. Acad. Sci. USA 74, 2662-2663 (1977)

12. Kiskis, J.: Phys. Rev. D 15, 2329-2336 (1977)

13. Brown, L., Carlitz, R., Lee, C.: Phys. Rev. D 16, 417-422 (1977)

14. Jackiv, R., Rebbi, C. : Phys. Rev. D 16, 1052-1060 (1977)

15. Bernard, C., Christ, N., Guth, A., Weinberg, E.: Phys. Rev. D 16, 2967-2977 (1977)

16. Nielsen, N.: Schroer, B. : Nucl. Phys. B 127, 493-508 (1977)

17. Atiyah, M., Ward, R. : Commun. math. Phys. 55, 117-124 (1977)

18. Atiyah, M., Hitchin, N., Drinfeld, V., Manin, Yu. : Oxford (preprint)

19. Drinfeld, V., Manin, Yu. : Func. Anal. i Prilozhen. (to appear) Uspehi Math. Nauk (to appear)

20. Stora, R.: In : Lectures at the International School of Math. Physics, Erice 1977

21. Mayer, M.: In : Proceedings of the 1977 Conference on Differential Geometrical Methods in Math. Physics. Bleuler, K., Reetz, A. (eds.). Lecture Notes in Mathematics. Berlin, Heidelberg, New York: Springer 1978

22. Forger, M.: Berlin (preprint)

23. Jackiw, R., Nohl, C., Rebbi, C.: MIT preprint (to be published by Plenum Press)

24. Schwarz, A.: Lett. Math. Phys. 2, 201-205 (1978)

25. Ray, D., Singer, I.: Adv. Math. 7, 145-210 (1971)

26. Schwarz, A.: Lett. Math. Phys. 2, 247-252 (1978)

27. Schwarz, A.: Commun. math. Phys. (submitted)

28. Schwarz, A.: Commun. math. Phys. 56, 79-86 (1977)

29. Frolov, I., Schwarz, A.: Phys. Lett. (submitted)

30. Yoneya, T.: Phys. Lett. B 71, 407-411 (1977)

31. Atiyah, M., Singer, I.: Ann. Math. 87, 484-530 (1968)

32. Palais, R.: Seminar on the Atiyah-Singer index theorem, Princeton, 1965

33. Atiyah, M., Bott, R., Patodi, V.: Inventiones Math. 19, 279-330 (1973)

34. Seeley, R.: Proc. Symp. Pure Math. 10, 288-307 (1971) 
35. Greub, W., Halperin, S., Vanstone, R.: Connections, curvature and cohomology. New York: Academic Press, Vol. I, 1972; Vol. II, 1973

36. Berezin, F.: The method of second quantization. New York: Academic Press 1966

37. Wilson, K.: Phys. Rev. D 10, 2445-2459 (1974)

38. Dynkin, E. : Matem. sbornik 35, 129-173 (1954) (in Russian)

39. Schwinger, J.: Particles, sources and fields. Reading, Massachusetts: Addison-Wesley 1970

40. Ray, D., Singer, I.: Ann. Math. 98, 154-177 (1973)

41. Hsiang, W. J.: In: Proceedings of Conference on Compact Transformation Groups. Berlin, Heidelberg, New York: Springer 1967

42. Frolov, I., Schwarz, A.: Phys. Lett. B (submitted)

43. Hartshorne, R. : Commun. math. Phys.

44. Frolov, I., Schwarz, A.: ZETF Lett. 28, 273-276 (1978)

45. Romanov, V., Schwarz, A.: Teor. Mat. Fiz. (submitted)

46. Gross, D., Wilczek, F.: Phys. Rev. Lett. 30, 1343-1346 (1973)

Communicated by R. Stora

Received July 18, 1978 